\title{
Structures on the conformal manifold in six dimensional theories
}

\author{
Hugh Osborn ${ }^{a}$ and Andreas Stergiou ${ }^{b}$ \\ ${ }^{a}$ Department of Applied Mathematics and Theoretical Physics, \\ Wilberforce Road, Cambridge CBЗ OWA, U.K. \\ ${ }^{b}$ Department of Physics, Yale University, \\ New Haven, CT 06520, U.S.A. \\ E-mail: ho@damtp.cam.ac.uk, andreas.stergiou@yale.edu
}

ABSTRACT: The tensors which may be defined on the conformal manifold for six dimensional CFTs with exactly marginal operators are analysed by considering the response to a Weyl rescaling of the metric in the presence of local couplings. It is shown that there are three symmetric two index tensors only one of which satisfies any positivity conditions. The general results are specialised to the six dimensional conformal theory defined by free two-forms and also to the interacting scalar $\phi^{3}$ theory at two loops which preserves conformal invariance to this order. All three two index tensor contributions are present.

KEYWords: Conformal and W Symmetry, Renormalization Group

ArXiv EPrint: 1501.01308 


\section{Contents}

1 Introduction 1

2 Response to Weyl rescalings for CFTs 4

$\begin{array}{lll}3 & \text { Two-forms } & 8\end{array}$

4 Calculations in scalar $\phi^{3}$ theory $\quad 11$

5 Positivity constraints 13

$\begin{array}{lll}6 & \text { Discussion } & 16\end{array}$

$\begin{array}{ll}\text { A Conformal tensors, invariants and operators } & 19\end{array}$

B Expansion of six dimensional dilaton action $\quad 24$

$\begin{array}{ll}\text { C Fermions } & 24\end{array}$

$\begin{array}{ll}\text { D Two-forms } & 25\end{array}$

\section{Introduction}

If conformal field theories have exactly marginal operators there is a conformal manifold parameterised by the couplings for the marginal operators. In two and four dimensions CFTs with associated conformal manifolds are not uncommon, at least with $\mathcal{N}=1$ supersymmetry [1]. The situation is much less clear in higher dimensions; whether any non trivial CFTs with marginal operators exist in six dimensions remains doubtful but not inconceivable [2]. Here we aim to extend some results obtained in two and four dimensions to the significantly more complicated case of six.

To this end we consider the response of a CFT extended to a curved space background to a Weyl rescaling of the metric $\gamma_{\mu \nu}$. In general, CFTs are invariant under Weyl rescalings of the background metric, $\gamma_{\mu \nu} \rightarrow e^{2 \sigma} \gamma_{\mu \nu}$, up to a finite sum of local contributions formed from curvature tensors and $\sigma$, with coefficients commonly referred to as central charges. In two dimensions there is just the Virasoro central charge $c$, so that the trace of the energy momentum tensor is proportional to $c R$, with $R$ the scalar curvature which is equal to the two dimensional Euler density $E_{2}$. In four dimensions there are just two coefficients $c, a$, which are related to the square of the Weyl tensor and the four dimensional Euler density $E_{4}$. These results for CFTs on curved backgrounds may be used to construct effective field theories for a dilaton $\tau$, with terms $\mathrm{O}\left(\tau^{2}\right)$ in two dimensions, and $\mathrm{O}\left(\tau^{3}, \tau^{4}\right)$ in four dimensions, which survive on reduction to flat space and are proportional to $c, a$ 
respectively. By considering dilaton scattering in four dimensions the crucial positivity constraints allowing arguments for an irreversible RG flow between UV and IR fixed points have been obtained $[3,4]$.

For CFTs with a conformal manifold it is convenient to allow the couplings $g^{I}$ for the marginal operators to be local or $x$-dependent. The couplings can then be treated as sources for the marginal operators. In that case there are additional local contributions under a Weyl rescaling depending on derivatives of $g^{I}$. Such terms are restricted by power counting. In two dimensions this procedure generates a unique two index tensor $g_{I J}$ on the conformal manifold, while in four dimensions a four index tensor is present also. In two dimensions $g_{I J}$ is identical with the metric defined by Zamolodchikov [5] in terms of the two point functions for the scalar operators coupled to $g^{I}$ and which for unitary theories is necessarily positive. A similar result applies in the four dimensional case so the corresponding metric is again positive.

Away from a conformal critical point the response to Weyl rescalings with local couplings must satisfy Wess-Zumino consistency conditions stemming from the fact that the Weyl group is Abelian. The resulting equations relate the RG flow of the central charge $c$ in two and $a$ in four dimensions to the corresponding $g_{I J}$. For positive $g_{I J}$ the RG flow is irreversible [6-8]. In two dimensions this approach is equivalent to the Zamolodchikov $c$-theorem. In four dimensions the metric is necessarily positive in the neighbourhood of a fixed point, but unlike two dimensions there is no simple general non perturbative argument, although arguments based on dilaton effective actions can be applied [8]. For renormalisable quantum field theories in four dimensions the metric and related quantities may be calculated perturbatively in terms of the vacuum amplitude, most directly with a curved space background and using local couplings at two loops [9], but also just restricting to flat space at three loops $[7,10,11]$.

It is natural to consider extensions to higher dimensions, in particular six. The dilaton effective action was constructed in [12] and also [14, 15]. The local RG approach was also extended to six dimensions in [16]. The number of contributions which it is necessary to consider increases significantly; in the approach followed in [16] there are $\mathrm{O}(100)$ different consistency conditions to be analysed. Due to complications arising from the analytic structure of $3 \rightarrow 3$ amplitudes there is no derivation of irreversibility of RG flow along the same lines as that applied in four dimensions [12], and recently a two loop calculation in six dimensional $\phi^{3}$ theory showed that the metric relevant for RG flow was not positive in this theory [17].

In this paper we endeavour to understand further the complications arising in six dimensions by considering a six dimensional conformal field theory with exactly marginal operators. The approach followed here, based on assuming local couplings for all marginal operators and considering the response to Weyl rescalings of the metric, defines various tensors on any conformal manifold. An infinitesimal Weyl rescaling determines the trace of the energy momentum tensor. As is well known, in six dimensions on a curved background with fixed couplings and neglecting scheme dependent contributions, this is expressible in terms of three scale dimension six Weyl invariants, with coefficients $c_{1}, c_{2}, c_{3}$, and the topological Euler density $E_{6}$, with coefficient $a[18,19]$. Thus $c_{1}, c_{2}, c_{3}, a$ may be regarded 
as the central charges in six dimensions, corresponding to the two dimensional $c$ and four dimensional $c, a$. With local couplings to marginal operators it is further possible to obtain three rank two symmetric tensors, as well as rank four and rank six tensors. One rank two symmetric tensor can be related to the two point function for marginal operators and is therefore positive. This may then be taken as a metric for the conformal manifold. However, contrary to the case in two and four dimensions, this is not the tensor that features in the equation for the RG flow of $a$. The additional symmetric tensors present in six dimensions are constructed in terms of the Weyl tensor and so are absent in any conformally flat space.

In the next section we review the response of a CFT containing exactly marginal operators in four dimensions and then consider the extension to six. In six dimensions it is necessary to consider Weyl transformations which are rather more involved than in four. Besides the Weyl tensor the results can be expressed more simply in terms a basis involving the Cotton and Bach tensors [22]. Their definitions and some basic properties are reviewed in appendix A. It is also necessary to consider various conformally covariant differential operators which extend the conformal Laplacian $\Delta_{2}=-\nabla^{2}+\xi R$, where $\xi=(d-2) / 4(d-1)$ with $d$ the spacetime dimension. In four dimensions the results involve $\Delta_{4}$, the conformal extension of $\left(\nabla^{2}\right)^{2}$, while in six dimensions it is necessary to consider the Branson operator $\Delta_{6}[50]$ whose leading term is $-\left(\nabla^{2}\right)^{3}$.

As an illustration of these results we consider in section 3 the conformal theory in six dimensions which is obtained from the quantum field theory of free two-forms. In this case we may introduce a local coupling in the action as $1 / g^{2}$ which acts as a source for the dimension six scalar operator formed by the gauge invariant classical Lagrangian density. After suitable gauge fixing we determine the one loop anomalous contributions under a Weyl rescaling of the metric, extending the results in [20] to include contributions involving derivatives of $g$. The results fit the general structure determined in section 2 .

In section 4 results obtained from calculations at two loops for $\phi^{3}$ theory on a curved background with local couplings are also presented. This theory has non zero $\beta$-functions and conformal invariance is broken but perturbative calculations should satisfy the constraints obtained in section 2 to lowest order. We also present results for the central charges $c_{1}, c_{2}, c_{3}, a$ to $\mathrm{O}\left(g^{2}\right)$. To ensure that the results are compatible with the general analysis it is necessary to ensure when using dimensional regularisation that the one loop counterterms are such as to ensure the initial free theory is conformal away from $d=6$. Although $\phi^{3}$ theory is problematic, since it lacks a minimum energy ground state, we assume it may be stabilised by a small $\phi^{4}$ term and that it may then still be used to define an effective conformal theory, at least to leading order.

We also consider in section 5 some positivity conditions which are obtained by relations to two point functions. These serve as a check on the results for $c_{3}$ which is related to the energy momentum tensor two point function and also a two index tensor on the space of marginal couplings which is related to the two point function for the exactly marginal dimension six scalar operators. The coefficients $c_{1}, c_{2}$ as well as $c_{3}$ determine the energy momentum tensor three point function. This also satisfies positivity restrictions related to the energy flux at infinity [21] and these are shown to be satisfied to lowest order beyond free theory by $\phi^{3}$ theory. 
Various details are contained in four appendices. In appendix A we present a detailed summary of results for conformal tensors, the Weyl, Cotton and Bach tensors, and also differential operators which transform nicely under Weyl rescaling of the metric and are relevant for our calculations. We also give an expression for the coincident limit of the Seeley-DeWitt coefficient $a_{3}$, which determines the one loop results, in terms of the basis of conformal tensors. In appendix $\mathrm{B}$ we describe briefly the six dimensional results obtained by integrating the infinitesimal Weyl rescaling of the metric. Appendices $\mathrm{C}$ and $\mathrm{D}$ contain the detailed results necessary to calculate the coincident limit of $a_{3}$ for fermions and two-forms respectively.

\section{Response to Weyl rescalings for CFTs}

In general the vacuum functional $W$, depending on the metric and couplings, for a CFT responds to an infinitesimal Weyl rescaling, $\delta_{\sigma} \gamma_{\mu \nu}=2 \sigma \gamma_{\mu \nu}$, in even $d$ dimensions according to

$$
(4 \pi)^{\frac{d}{2}} \delta_{\sigma} W=\int \mathrm{d}^{d} x \sqrt{-\gamma} \sigma L_{d}
$$

with $L_{d}$ a local scalar of dimension $d$ formed from the metric, the couplings and derivatives. In general $L_{d}$ is constrained by the integrability conditions following from $\left(\delta_{\sigma} \delta_{\sigma^{\prime}}-\right.$ $\left.\delta_{\sigma^{\prime}} \delta_{\sigma}\right) W=0$. We initially consider solutions such that

$$
\delta_{\sigma} L_{d}+d \sigma L_{d}=\nabla_{\mu}\left(X_{d}^{\mu \nu} \partial_{\nu} \sigma\right), \quad X_{d}^{\mu \nu}=X_{d}^{\nu \mu} .
$$

We assume that in (2.1) $L_{d}$ has the freedom

$$
L_{d} \sim L_{d}+\nabla_{\mu} \nabla_{\nu} Z_{d}^{\mu \nu}
$$

since such contributions can in general be cancelled by local contributions to $W$. For variations (2.3) compatible with (2.2) then

$$
X_{d}^{\mu \nu} \sim X_{d}^{\mu \nu}+2 Z_{d}^{\mu \nu}-\gamma^{\mu \nu} Z_{d}^{\lambda} \lambda \quad \text { if } \quad \delta_{\sigma} Z_{d}^{\mu \nu}+d \sigma Z_{d}^{\mu \nu}=0 .
$$

Under a finite rescaling (2.1) extends to

$$
(4 \pi)^{\frac{d}{2}}\left(W\left[e^{2 \sigma} \gamma_{\mu \nu}\right]-W\left[\gamma_{\mu \nu}\right]\right)=\int \mathrm{d}^{d} x \sqrt{-\gamma} \mathcal{L}_{d}(\sigma)
$$

where $\mathcal{L}_{d}(\sigma)$ is obtained by a Taylor expansion,

$$
\begin{aligned}
\mathcal{L}_{d}(\sigma) & =\sigma L_{d}-\partial_{\mu} \sigma \partial_{\nu} \sigma \sum_{r \geq 0} \frac{1}{(r+2) !} X_{d, r}{ }^{\mu \nu}+\nabla_{\mu} J^{\mu}, \\
X_{d, r+1}{ }^{\mu \nu} & =\left(\delta_{\sigma}+d \sigma\right) X_{d, r}{ }^{\mu \nu}, \quad X_{d, 0}{ }^{\mu \nu}=X_{d}{ }^{\mu \nu},
\end{aligned}
$$

so that $X_{d, r}{ }^{\mu \nu}=\mathrm{O}\left(\sigma^{r}\right)$ and $J^{\mu}$ is arbitrary. The sum in (2.6) truncates after a finite number of terms.

Before proceeding to the six dimensional case we recapitulate previous results obtained in four dimensions [7]. The extra terms involving derivatives of the couplings depend on a 
symmetric two index tensor $g_{I J}$ and also a four index tensor $c_{I J K L}$. It is natural to express the contributions to $L_{4}$ using the Christoffel connection formed from $g_{I J}$,

$$
\Gamma_{J K}^{I}=\frac{1}{2} g^{I L}\left(\partial_{J} g_{L K}+\partial_{K} g_{L J}-\partial_{L} g_{J K}\right), \quad g^{I J}=\left(g^{-1}\right)^{I J} .
$$

We may also allow for a background gauge field $A_{\mu} \in \mathfrak{g}$ coupled to conserved currents. If $F_{\mu \nu}$ is the associated field strength, then

$$
\begin{aligned}
L_{4}= & c W^{\rho \mu \nu \lambda} W_{\rho \mu \nu \lambda}-a E_{4}-\frac{1}{4} \kappa_{a b} F_{a}{ }^{\mu \nu} F_{b \mu \nu} \\
& +\frac{1}{2} g_{I J} D^{2} g^{I} D^{2} g^{J}-g_{I J} \partial^{\mu} g^{I}\left(2 P_{\mu \nu}-\gamma_{\mu \nu} \hat{R}\right) \partial^{\nu} g^{J} \\
& +\frac{1}{2} c_{I J K L} \partial^{\mu} g^{I} \partial_{\mu} g^{J} \partial^{\nu} g^{K} \partial_{\nu} g^{L} .
\end{aligned}
$$

Here,

$$
D^{2} g^{I}=\nabla^{2} g^{I}+\Gamma_{J K}^{I} \partial^{\mu} g^{J} \partial_{\mu} g^{K},
$$

$E_{4}$ is the Euler density, given by (A.9) for $d=4$, and $P_{\mu \nu}$ and $\hat{R}$ are the Schouten tensor and its trace given by (A.1) for $d=4$. In (2.8) clearly $g_{I J}=g_{J I}, c_{I J K L}=c_{(I J)(K L)}=$ $c_{K L I J}$ and $\kappa_{a b}$ is a symmetric invariant bilinear form in a convenient basis $\left\{t_{a}\right\}$ for $\mathfrak{g}$ so that, for any $X \in \mathfrak{g}, X=X_{a} t_{a}$. If $\mathfrak{g}$ is simple then $\kappa_{a b} \rightarrow \kappa \delta_{a b}$. We may also extend $\partial_{\mu} g^{I} \rightarrow \partial_{\mu} g^{I}+A_{a \mu}\left(T_{a} g\right)^{I}$ but for simplicity we neglect such contributions here.

It is straightforward to check that (2.8) satisfies (2.2) with

$$
X_{4}{ }^{\mu \nu}=-8 a G_{4}{ }^{\mu \nu}+g_{I J}\left(2 \partial^{\mu} g^{I} \partial^{\nu} g^{J}-\gamma^{\mu \nu} \partial^{\lambda} g^{I} \partial_{\lambda} g^{J}\right),
$$

and $G_{4}{ }^{\mu \nu}$ as in (A.13) with $d=4$, so long as $a$ is constant. From (2.6) it is easy to see that

$$
X_{4,1}{ }^{\mu \nu}=16 a\left(\nabla^{\mu} \partial^{\nu} \sigma-\gamma^{\mu \nu} \nabla^{2} \sigma\right), \quad X_{4,2}{ }^{\mu \nu}=-16 a\left(2 \partial^{\mu} \sigma \partial^{\nu} \sigma+\gamma^{\mu \nu} \partial^{\lambda} \sigma \partial_{\lambda} \sigma\right) .
$$

Using (2.10), (2.11) in (2.6) gives

$$
\begin{aligned}
\mathcal{L}_{4}(\sigma)= & \sigma L_{4}+\frac{1}{2} g_{I J}\left(2 \partial^{\mu} g^{I} \partial^{\nu} g^{J}-\gamma^{\mu \nu} \partial^{\lambda} g^{I} \partial_{\lambda} g^{J}\right) \partial_{\mu} \sigma \partial_{\nu} \sigma \\
& +4 a\left(G_{4}{ }^{\mu \nu} \partial_{\mu} \sigma \partial_{\nu} \sigma+\nabla^{2} \sigma \partial^{\mu} \sigma \partial_{\mu} \sigma+\frac{1}{2}\left(\partial^{\mu} \sigma \partial_{\mu} \sigma\right)^{2}\right)
\end{aligned}
$$

which reproduces the well known results for the four dimensional dilaton effective action and the $\partial g$ terms calculated in [7].

In six dimensions we follow a similar route by determining the general form for $L_{6}$ satisfying (2.2). There are various contributions which may be analysed independently. For any six dimensional CFT in the absence of local couplings $L_{6}$ is given by just

$$
L_{6}^{R}=\sum_{i=1,2,3} c_{i} I_{i}+a E_{6},
$$

where an appropriate basis for the dimension six conformal scalars $I_{i}$, and also an explicit expression for the Euler density $E_{6}$, are given in appendix A. $I_{1}, I_{2}$ are the two independent 
scalars cubic in the Weyl tensor while $I_{3}=W^{\rho \mu \nu \lambda} \nabla^{2} W_{\rho \mu \nu \lambda}+\cdots$. Since $\delta_{\sigma} I_{i}+6 \sigma I_{i}=0$ and $\delta_{\sigma} E_{6}+6 \sigma E_{6}=24 \nabla_{\mu}\left(G_{6}{ }^{\mu \nu} \partial_{\nu} \sigma\right)$, with $G_{6}{ }^{\mu \nu}$ the six dimensional generalisation of the Einstein tensor, it is easy to verify that (2.13) satisfies (2.2) with

$$
X_{6}^{R \mu \nu}=24 a G_{6}{ }^{\mu \nu} .
$$

There are also three potential dimension six conformal scalars formed from $F_{\mu \nu}$ for which we may take

$$
\begin{aligned}
L_{6}^{F}= & -\frac{1}{4} \kappa_{a b}\left(F_{a}{ }^{\mu \nu}\left(\mathcal{D}^{2} F_{\mu \nu}\right)_{b}-4 \hat{R} F_{a}{ }^{\mu \nu} F_{b \mu \nu}\right)-2\left(\nabla_{\mu} \nabla_{\nu}+2 P_{\mu \nu}\right)\left(\kappa_{a b} F_{a}{ }^{\mu \lambda} F_{b}{ }^{\nu} \lambda\right) \\
& -\frac{1}{4} \hat{\kappa}_{a b} W_{\mu \nu \lambda \rho} F_{a}{ }^{\mu \nu} F_{b}{ }^{\lambda \rho}+\frac{1}{3} f_{a b c} F_{a}{ }^{\mu \nu} F_{b \nu \lambda} F_{c}{ }^{\lambda},
\end{aligned}
$$

with $\kappa_{a b}, \hat{\kappa}_{a b}$ symmetric invariant tensors and $f_{a b c}$ an antisymmetric invariant tensor.

For free scalars, fermions and also two-form gauge fields the coefficients $c_{i}, a$ were calculated in [20]. For scalars and fermions the results can be straightforwardly extended to include background gauge fields. For $t_{a}$ the real antisymmetric or anti-hermitian generators determining the gauge couplings to scalars or fermions, then, letting $\kappa_{a b}=$ $-\kappa \operatorname{tr}\left(t_{a} t_{b}\right), \hat{\kappa}_{a b}=-\hat{\kappa} \operatorname{tr}\left(t_{a} t_{b}\right), f_{a b c}=-f \operatorname{tr}\left(t_{[a} t_{b} t_{c]}\right)$, we have

\begin{tabular}{|lccccccc|}
\hline & $7 ! c_{1}$ & $7 ! c_{2}$ & $7 ! c_{3}$ & $7 ! a$ & $\kappa$ & $\hat{\kappa}$ & $f$ \\
\hline scalars & $-\frac{28}{3}$ & $\frac{5}{3}$ & 2 & $\frac{5}{9}$ & $\frac{1}{30}$ & $\frac{1}{18}$ & $\frac{1}{15}$ \\
fermions & $-\frac{896}{3}$ & -32 & 40 & $\frac{191}{9}$ & $\frac{16}{15}$ & $\frac{8}{9}$ & $\frac{52}{15}$ \\
two-forms & $-\frac{8008}{3}$ & $-\frac{2378}{3}$ & 180 & 442 & & & \\
\hline
\end{tabular}

Our main motivation in this paper is to consider contributions depending on derivatives of the couplings. We first consider terms which are the direct extension of the terms involving $g_{I J}$ in (2.8). This can be constructed starting from a leading contribution involving six derivatives

$$
S_{1}=\frac{1}{2} g_{I J} D^{\mu} D^{2} g^{I} D_{\mu} D^{2} g^{J}+\frac{1}{2} \mathcal{R}_{I K L J} D^{2} g^{I} \partial^{\mu} g^{K} \partial_{\mu} g^{L} D^{2} g^{J},
$$

with $D^{2} g^{I}$ defined as in (2.9) and

$$
D^{\mu} D^{2} g^{I}=\partial^{\mu} D^{2} g^{I}+\Gamma_{K L}^{I} \partial^{\mu} g^{K} D^{2} g^{L} .
$$

In (2.17) $\mathcal{R}_{I K L J}$ is the Riemann tensor defined as usual in terms of the Christoffel connection $\Gamma_{K L}^{I}$ in (2.7). The Weyl variation of $S_{1}$ gives

$$
\begin{aligned}
\delta_{\sigma} S_{1}+6 \sigma S_{1}= & -8 g_{I J} D^{2} g^{I} D^{\mu} \partial^{\nu} g^{J} \nabla_{\mu} \partial_{\nu} \sigma+3 g_{I J} D^{2} g^{I} D^{2} g^{J} \nabla^{2} \sigma \\
& +4 g_{I J} \partial^{\mu} g^{I} \partial^{\nu} g^{J}\left(\nabla_{\mu} \partial_{\nu} \nabla^{2} \sigma+8 \nabla_{\mu}\left(P_{\nu \lambda} \partial^{\lambda} \sigma\right)+2 \nabla_{\mu}\left(\hat{R} \partial_{\nu} \sigma\right)\right) \\
& -2 g_{I J} \partial^{\mu} g^{I} \partial_{\mu} g^{J}\left(\nabla^{2} \nabla^{2} \sigma+8 \nabla^{\nu}\left(P_{\nu \lambda} \partial^{\lambda} \sigma\right)+2 \nabla^{\nu}\left(\hat{R} \partial_{\nu} \sigma\right)\right) \\
& +\nabla_{\mu}\left(4 g_{I J} D^{2} g^{I} D^{\mu}\left(\partial^{\nu} g^{J} \partial_{\nu} \sigma\right)-3 g_{I J} D^{2} g^{I} D^{2} g^{J} \partial^{\mu} \sigma\right. \\
& \quad-4 g_{I J} \partial^{\mu} g^{I} \partial^{\nu} g^{J}\left(\partial_{\nu} \nabla^{2} \sigma+8 P_{\nu \lambda} \partial^{\lambda} \sigma+2 \hat{R} \partial_{\nu} \sigma\right) \\
& \left.+2 g_{I J} \partial^{\lambda} g^{I} \partial_{\lambda} g^{J}\left(\partial^{\mu} \nabla^{2} \sigma+8 P^{\mu \nu} \partial_{\nu} \sigma+2 \hat{R} \partial^{\mu} \sigma\right)\right)
\end{aligned}
$$


If we then add the four derivative term

$$
\begin{aligned}
S_{2}= & -4 g_{I J}\left(D^{2} g^{I} D^{\mu} \partial^{\nu} g^{J}+D^{\mu} \partial^{\nu} g^{I} D^{2} g^{J}\right) P_{\mu \nu}+3 g_{I J} D^{2} g^{I} D^{2} g^{J} \hat{R} \\
& +\nabla_{\mu}\left(4 P^{\mu \lambda} g_{I J} \partial_{\lambda} g^{I} D^{2} g^{J}-4 g_{I J} \partial^{\mu} g^{I} \partial^{\nu} g^{J} \partial_{\nu} \hat{R}+2 g_{I J} \partial^{\lambda} g^{I} \partial_{\lambda} g^{J} \partial^{\mu} \hat{R}\right),
\end{aligned}
$$

we may obtain

$$
\delta_{\sigma}\left(S_{1}+S_{2}\right)+6 \sigma\left(S_{1}+S_{2}\right)=g_{I J} \partial^{\mu} g^{I} \partial^{\nu} g^{J} A_{\mu \nu}+\nabla_{\mu}\left(X_{6}^{g \mu \nu} \partial_{\nu} \sigma\right),
$$

for

$$
\begin{aligned}
A_{\mu \nu}= & 4 \nabla_{\mu} \partial_{\nu} \nabla^{2} \sigma+32 \nabla^{\lambda}\left(P_{\lambda(\mu} \partial_{\nu)} \sigma\right)-16 \nabla^{\lambda}\left(P_{\mu \nu} \partial_{\lambda} \sigma\right)+16 \nabla_{(\mu}\left(P_{\nu) \lambda} \partial^{\lambda} \sigma\right) \\
& -8 \nabla_{(\mu}\left(\hat{R} \partial_{\nu)} \sigma\right) \\
& +\gamma_{\mu \nu}\left(-2 \nabla^{2} \nabla^{2} \sigma-8 \nabla^{\mu}\left(P_{\mu \nu} \partial^{\nu} \sigma\right)+4 \nabla^{\mu}\left(\hat{R} \partial_{\mu} \sigma\right)\right)
\end{aligned}
$$

and

$$
\begin{aligned}
X_{6}^{g \mu \nu}= & 4 g_{I J} D^{2} g^{I} D^{\mu} \partial^{\nu} g^{J}-3 \gamma^{\mu \nu} g_{I J} D^{2} g^{I} D^{2} g^{J} \\
& +8 P^{\mu \nu} g_{I J} \partial^{\lambda} g^{I} \partial_{\lambda} g^{J}-16\left(P^{\mu \lambda} g_{I J} \partial_{\lambda} g^{I} \partial^{\nu} g^{J}+g_{I J} \partial^{\mu} g^{I} \partial_{\lambda} g^{J} P^{\lambda \nu}\right) \\
& +16 g_{I J} \partial^{\mu} g^{I} \partial^{\nu} g^{J} \hat{R}+\gamma^{\mu \nu}\left(16 g_{I J} \partial^{\lambda} g^{I} \partial^{\rho} g^{J} P_{\lambda \rho}-8 \partial^{\lambda} g^{I} \partial_{\lambda} g^{J} \hat{R}\right),
\end{aligned}
$$

where $P_{\mu \nu}, \hat{R}$ are given by (A.1) with $d=6$. The remaining $A_{\mu \nu}$ terms in (2.21) may be cancelled by taking

$$
\begin{aligned}
S_{3}= & 4 g_{I J} \partial^{\mu} g^{I} \partial^{\nu} g^{J}\left(B_{\mu \nu}+6 P_{\mu \lambda} P_{\nu}{ }^{\lambda}-4 P_{\mu \nu} \hat{R}+\nabla_{\mu} \partial_{\nu} \hat{R}\right) \\
& -2 g_{I J} \partial^{\mu} g^{I} \partial_{\mu} g^{J}\left(2 P_{\rho \lambda} P^{\rho \lambda}-2 \hat{R}^{2}+\nabla^{2} \hat{R}\right) .
\end{aligned}
$$

Hence, we may satisfy (2.2) for $d=6$ by taking

$$
\begin{aligned}
L_{6}^{g}= & -S_{1}-S_{2}-S_{3} \\
& +g_{1, I J} \partial^{\mu} g^{I} \partial^{\nu} g^{J} W_{\mu \lambda \rho \omega} W_{\nu}^{\lambda \rho \omega}+g_{2, I J} \partial^{\mu} g^{I} \partial_{\mu} g^{J} W_{\nu \lambda \rho \omega} W^{\nu \lambda \rho \omega} .
\end{aligned}
$$

The terms involving $g_{I J}$ are a natural generalisation of the unique $L_{4}^{g}$, implicitly defined by (2.8), and $L_{2}^{g}=-\frac{1}{2} g_{I J} \partial^{\mu} g^{I} \partial_{\mu} g^{J}$. The sign is chosen so as to ensure later that $g_{I J}$ is positive in unitary theories. In six dimensions there are further possibilities involving rank two tensors which are formed in terms of the Weyl tensor, as included in (2.25). For these terms (2.2) becomes essentially trivial.

Further contributions to $L_{6}$ involve at least four $g$ 's with derivatives. To construct these we first consider

$$
\begin{aligned}
T_{1}= & j_{1, I J K L} \frac{1}{2}\left(\nabla_{\rho} h^{I J \mu \rho} \nabla^{\lambda} h_{\mu \lambda}^{K L}-\nabla^{\lambda} h^{I J \mu \nu} \nabla_{\lambda} h_{\mu \nu}^{K L}\right) \\
& +j_{2, I J K L} \frac{1}{2} \partial^{\lambda}\left(\partial^{\mu} g^{I} \partial_{\mu} g^{J}\right) \partial_{\lambda}\left(\partial^{\nu} g^{K} \partial_{\nu} g^{L}\right) \\
& +j_{3, I J K L}\left(\nabla^{\mu} \partial^{\nu} g^{I} \nabla_{\mu} \partial_{\nu} g^{J}-\frac{1}{4} \nabla^{2} g^{I} \nabla^{2} g^{J}\right) \partial^{\rho} g^{K} \partial_{\rho} g^{L}
\end{aligned}
$$

for $h^{I J}{ }_{\mu \nu}$ symmetric and traceless,

$$
h_{\mu \nu}^{I J}=\partial_{(\mu} g^{I} \partial_{\nu)} g^{J}-\frac{1}{6} \gamma_{\mu \nu} \partial^{\lambda} g^{I} \partial_{\lambda} g^{J},
$$


and $j_{i, I J K L}=j_{i,(I J)(K L)}=j_{i, K L I J}$. In this case

$$
\begin{aligned}
\delta_{\sigma} T_{1}+6 \sigma T_{1}= & \partial_{\lambda} \sigma j_{1, I J K L}\left(2 \nabla_{\mu}\left(h^{I J \lambda \nu} h^{K L \mu}{ }_{\nu}\right)+\partial^{\lambda}\left(h^{I J \mu \nu} h^{K L}{ }_{\mu \nu}\right)\right) \\
& -\partial_{\lambda} \sigma\left(j_{2, I J K L}+j_{3, I J K L}\right) \partial^{\lambda}\left(\partial^{\mu} g^{I} \partial_{\mu} g^{J} \partial^{\nu} g^{K} \partial_{\nu} g^{L}\right) .
\end{aligned}
$$

Terms involving two derivatives of $\sigma$ may be cancelled by

$$
\begin{aligned}
T_{2}= & -j_{1, I J K L}\left(2 P_{\mu \nu} h^{I J \mu \lambda} h_{\lambda L}^{K L \nu}+\hat{R} h^{I J \mu \nu} h_{\mu \nu}^{K L}\right) \\
& +\left(j_{2, I J K L}+j_{3, I J K L}\right) \hat{R} \partial^{\mu} g^{I} \partial_{\mu} g^{J} \partial^{\nu} g^{K} \partial_{\nu} g^{L} \\
& -\partial_{M} j_{1, I J K L}\left(h^{I J \mu \lambda} h^{K L}{ }_{\mu \rho} \nabla^{\rho} \partial_{\lambda} g^{M}-\frac{1}{2} h^{I J \mu \nu} h^{K L}{ }_{\mu \nu} \nabla^{2} g^{M}\right) \\
& -\frac{1}{4} \partial_{M}\left(j_{2, I J K L}+j_{3, I J K L}\right) \partial^{\mu} g^{I} \partial_{\mu} g^{J} \partial^{\nu} g^{K} \partial_{\nu} g^{L} \nabla^{2} g^{M} .
\end{aligned}
$$

Hence

$$
L_{6}^{j}=T_{1}+T_{2}+j_{4, I J K L} W_{\mu \lambda \nu \rho} h^{I J \mu \nu} h^{K L \lambda \rho}
$$

satisfies (2.2) with

$$
\begin{aligned}
X_{6}^{j \mu \nu}= & j_{1, I J K L}\left(2 h^{I J \mu \lambda} h_{\lambda L \nu}^{K L}+\gamma^{\mu \nu} h^{I J \lambda \rho} h_{\lambda \rho}^{K L}\right) \\
& -\gamma^{\mu \nu}\left(j_{2, I J K L}+j_{3, I J K L}\right) \partial^{\lambda} g^{K} \partial_{\lambda} g^{J} \partial^{\rho} g^{K} \partial_{\rho} g^{L} .
\end{aligned}
$$

In (2.30) we have allowed for a possible trivial term involving the Weyl tensor. If in (2.26) $\nabla_{\lambda} h^{K L}{ }_{\mu \nu} \rightarrow D_{\lambda} h^{K L}{ }_{\mu \nu}$ and similarly $\partial_{\lambda}\left(\partial^{\nu} g^{K} \partial_{\nu} g^{L}\right) \rightarrow D_{\lambda}\left(\partial^{\nu} g^{K} \partial_{\nu} g^{L}\right), \nabla^{\mu} \partial^{\nu} g^{I} \rightarrow D^{\mu} \partial^{\nu} g^{I}$, $\nabla^{2} g^{I} \rightarrow D^{2} g^{I}$, with $D_{\lambda}$ the covariant derivative including the the Christoffel connection (2.7), then correspondingly in (2.29) $\partial_{M} j_{i, I J K L} \rightarrow D_{M} j_{i, I J K L}$ with $D_{M}$ the covariant extension of $\partial_{M}$.

The remaining potential contribution to $L_{6}$ involves six $g$ 's with derivatives,

$$
L_{6}^{k}=\frac{1}{2} k_{I J K L M N} \partial^{\mu} g^{I} \partial_{\mu} g^{J} \partial^{\nu} g^{K} \partial_{\nu} g^{L} \partial^{\omega} g^{M} \partial_{\omega} g^{N},
$$

defining a rank six tensor with appropriate symmetries.

\section{Two-forms}

In six dimensions there are three free conformal field theories. In four dimensions with abelian gauge fields it is still possible to determine the leading one loop contribution to the metric on the conformal manifold. Here we describe the analogous calculation in six dimensions following the approach described in [24] and extending the six dimensional results in [20].

For a two-form $B_{\mu \nu} \in \Omega^{(2)}$, where $\Omega^{(n)}$ is the space of $n$-forms comprised of antisymmetric $n$-index tensors, the starting Lagrangian is just ${ }^{1}$

$$
\mathscr{L}=-\frac{1}{12 g^{2}}(\mathrm{~d} B)^{\mu \nu \omega}(\mathrm{d} B)_{\mu \nu \omega} .
$$

\footnotetext{
${ }^{1}$ The exterior derivative $\mathrm{d}: \Omega^{(n)} \rightarrow \Omega^{(n+1)}$, is defined so that $(\mathrm{d} F)_{\mu_{1} \ldots \mu_{n+1}}=(n+1) \partial_{\left[\mu_{1}\right.} F_{\left.\mu_{2} \ldots \mu_{n+1}\right]}$ for $F_{\mu_{1} \ldots \mu_{n}} \in \Omega^{(n)}$ and is independent of the metric. The adjoint $\delta: \Omega^{(n)} \rightarrow \Omega^{(n-1)}$ is correspondingly given by $(\delta F)_{\mu_{1} \ldots \mu_{n-1}}=-\frac{1}{\sqrt{-\gamma}} \gamma_{\mu_{1} \nu_{1}} \cdots \gamma_{\mu_{n-1} \nu_{n-1}} \partial_{\omega}\left(\sqrt{-\gamma} \gamma^{\omega \lambda} \gamma^{\nu_{1} \rho_{1}} \cdots \gamma^{\nu_{n-1} \rho_{n-1}} F_{\lambda \rho_{1} \ldots \rho_{n-1}}\right)=-\nabla^{\lambda} F_{\lambda \mu_{1} \ldots \mu_{n-1}}$. Of course $\mathrm{d}^{2}=\delta^{2}=0$.
} 
This is invariant under gauge transformations $B_{\mu \nu} \rightarrow B_{\mu \nu}+(\mathrm{d} A)_{\mu \nu}, A_{\mu} \in \Omega^{(1)}$. It is convenient here to add the covariant Feynman gauge fixing term,

$$
\mathscr{L}_{\text {g.f. }}=-\frac{g^{2}}{2}\left(\delta\left(\frac{1}{g^{2}} B\right)\right)^{\mu}\left(\delta\left(\frac{1}{g^{2}} B\right)\right) \mu .
$$

Rescaling $B_{\mu \nu} \rightarrow g B_{\mu \nu}$ the quantum theory is defined in terms of the functional determinants of the Laplacians

$$
\Delta^{(n)}=\delta^{\prime} \mathrm{d}^{\prime}+\mathrm{d}^{\prime} \delta^{\prime}: \Omega^{(n)} \rightarrow \Omega^{(n)}, \quad \mathrm{d}^{\prime}=\frac{1}{g} \mathrm{~d} g, \quad \delta^{\prime}=g \delta \frac{1}{g},
$$

so that $[20]$

$$
W=-\frac{1}{2} \ln \operatorname{Det} \Delta^{(2)}+\ln \operatorname{Det} \Delta^{(1)}-\frac{3}{2} \ln \operatorname{Det} \Delta^{(0)} .
$$

$\Delta^{(1)}$ is related to a fermionic vector ghost and $\Delta^{(0)}$ to a bosonic scalar ghost; the degrees of freedom in $d$ dimensions are then $\frac{1}{2} d(d-1)-2 d+3=\frac{1}{2}(d-2)(d-3)$.

Continuing to a Euclidean metric the functional determinant of an elliptic differential operator $\Delta$ may be defined in terms of the heat kernel by

$$
-\ln \operatorname{Det} \Delta=\zeta_{\Delta^{\prime}}^{\prime}(0), \quad \zeta_{\Delta}(s)=\frac{1}{\Gamma(s)} \int_{0}^{\infty} \mathrm{d} \tau \tau^{s-1} \operatorname{Tr}\left(e^{-\tau \Delta}\right) .
$$

Under Weyl rescaling of the metric, for $F_{\mu_{1} \ldots \mu_{n}}$ an $n$-form, $\delta_{\sigma}(\mathrm{d} F)_{\mu_{1} \ldots \mu_{n+1}}=0$, whereas $\delta_{\sigma}(\delta F)_{\mu_{1} \ldots \mu_{n-1}}=-2(d-n+1) \sigma(\delta F)_{\mu_{1} \ldots \mu_{n-1}}+2(d-n)(\delta \sigma F)_{\mu_{1} \ldots \mu_{n-1}}$. Hence, with $d=6$,

$$
\begin{aligned}
& \delta_{\sigma} \Delta^{(2)}=-2 \sigma \Delta^{(2)}+2 \sigma \mathrm{d}^{\prime} \delta^{\prime}+2 \mathrm{~d}^{\prime} \delta^{\prime} \sigma-4 \mathrm{~d}^{\prime} \sigma \delta^{\prime}, \\
& \delta_{\sigma} \Delta^{(1)}=-2 \sigma \Delta^{(1)}+2 \sigma \mathrm{d}^{\prime} \delta^{\prime}+4 \mathrm{~d}^{\prime} \delta^{\prime} \sigma-6 \mathrm{~d}^{\prime} \sigma \delta^{\prime}+2 \delta^{\prime} \sigma \mathrm{d}^{\prime}-2 \sigma \delta^{\prime} \mathrm{d}^{\prime}, \\
& \delta_{\sigma} \Delta^{(0)}=-2 \sigma \Delta^{(0)}-4 \sigma \delta^{\prime} \mathrm{d}^{\prime}+4 \delta^{\prime} \sigma \mathrm{d}^{\prime} .
\end{aligned}
$$

Using relations such as $\mathrm{d}^{\prime} \Delta^{(1)}=\Delta^{(2)} \mathrm{d}^{\prime}$ we may obtain

$$
\begin{aligned}
& \delta_{\sigma}\left(\operatorname{Tr}_{\Omega^{(2)}}\left(e^{-\tau \Delta^{(2)}}\right)-2 \operatorname{Tr}_{\Omega^{(1)}}\left(e^{-\tau \Delta^{(1)}}\right)+3 \operatorname{Tr}_{\Omega^{(0)}}\left(e^{-\tau \Delta^{(0)}}\right)\right) \\
& =-2 \tau \frac{\mathrm{d}}{\mathrm{d} \tau}\left(\operatorname{Tr}_{\Omega^{(2)}}\left(\sigma e^{-\tau \Delta^{(2)}}\right)-2 \operatorname{Tr}_{\Omega^{(1)}}\left(\sigma e^{-\tau \Delta^{(1)}}\right)+3 \operatorname{Tr}_{\Omega^{(0)}}\left(\sigma e^{-\tau \Delta^{(0)}}\right)\right),
\end{aligned}
$$

so that from (3.4) and (3.5)

$$
\delta_{\sigma} W=\left.\left(\operatorname{Tr}_{\Omega^{(2)}}\left(\sigma e^{-\tau \Delta^{(2)}}\right)-2 \operatorname{Tr}_{\Omega^{(1)}}\left(\sigma e^{-\tau \Delta^{(1)}}\right)+3 \operatorname{Tr}_{\Omega^{(0)}}\left(\sigma e^{-\tau \Delta^{(0)}}\right)\right)\right|_{\tau^{0}},
$$

with $\left.\right|_{\tau^{0}}$ denoting the $\tau^{0}$ term in the Laurent expansion in $\tau$.

In each case the Laplacians defined in (3.3) have the form

$$
\Delta=-\mathcal{D}^{2}+2 \hat{R} \mathbb{1}_{V}+Y_{\Delta}
$$

for $\Delta: V \rightarrow V$ and $\mathcal{D}_{\mu}=\nabla_{\mu}+A_{\mu}$ with $A_{\mu}$ an appropriate connection on $V$. For such elliptic operators the associated heat kernel $K_{\Delta}(x, y ; \tau)$, corresponding to $e^{-\tau \Delta}$, has 
the well known expansion $(4 \pi \tau)^{\frac{1}{2} d} K_{\Delta}(x, x ; \tau) \sim \sum_{n \geq 0} a_{\Delta, n} \mid(x) \tau^{n}$ with $a_{\Delta, n} \mid$ the diagonal Seeley-DeWitt coefficients. Hence for $d=6$

$$
\left.(4 \pi)^{3} \operatorname{Tr}_{V}\left(\sigma e^{-\tau \Delta}\right)\right|_{\tau^{0}}=\int \mathrm{d}^{6} x \sqrt{\gamma} \sigma \operatorname{tr}_{V}\left(a_{\Delta, 3} \mid\right),
$$

with $\operatorname{tr}_{V}$ the matrix trace and

$$
\begin{aligned}
7 ! \operatorname{tr}_{V} & \left(a_{\Delta, 3} \mid\right)=\operatorname{dim} V\left(\frac{5}{9} E_{6}-\frac{28}{3} I_{1}+\frac{5}{3} I_{2}+2 I_{3}\right) \\
+ & 14\left(3 \operatorname{tr}_{V}(\hat{I})+5 W_{\mu \nu \lambda \rho} \operatorname{tr}_{V}\left(F^{\mu \nu} F^{\lambda \rho}\right)-8 \operatorname{tr}_{V}\left(F^{\mu \nu} F_{\nu \lambda} F^{\lambda}{ }_{\mu}\right)\right) \\
- & 7 ! \operatorname{tr}_{V}\left(\frac{1}{6} Y_{\Delta}^{3}+\frac{1}{12} Y_{\Delta} \Delta_{2} Y_{\Delta}+\frac{1}{180} W^{\rho \mu \nu \lambda} W_{\rho \mu \nu \lambda} Y_{\Delta}+\frac{1}{12} F^{\mu \nu} F_{\mu \nu} Y_{\Delta}\right) \\
+ & 7 ! \nabla_{\mu} \nabla_{\nu} Z_{\Delta}{ }^{\mu \nu} .
\end{aligned}
$$

Here $\operatorname{tr}_{V}(\hat{I})=\operatorname{tr}_{V}\left(F^{\mu \nu} \mathcal{D}^{2} F_{\mu \nu}\right)+\cdots$ is a dimension six conformal scalar formed from $F_{\mu \nu}$, and $\Delta_{2}=-\mathcal{D}^{2}+2 \hat{R}$ with $\mathcal{D}_{\mu} Y_{\Delta}=\partial_{\mu} Y_{\Delta}+\left[A_{\mu}, Y_{\Delta}\right]$. For zero-forms $F_{\lambda \rho} \rightarrow 0$ while acting on one-forms $A_{\mu}, F_{\lambda \rho} \rightarrow R_{\mu}{ }^{\mu^{\prime}}{ }_{\lambda \rho}$ and on two-forms $B_{\mu \nu}, F_{\lambda \rho} \rightarrow 2 \delta_{[\mu}{ }^{\left[\mu^{\prime}\right.} R_{\nu]}{ }^{\left.\nu^{\prime}\right]} \lambda \rho$. An explicit form for $Z_{\Delta}{ }^{\mu \nu}$ in (3.11) is given in appendix A.

For the operators $\Delta^{(n)}$, and letting $Y_{\Delta^{(n)}} \equiv Y_{n}$,

$$
\begin{aligned}
Y_{0} & =-2 \hat{R}+U, \\
Y_{1 \mu}{ }^{\mu^{\prime}} & =(-\hat{R}+U) \delta_{\mu}^{\mu^{\prime}}+4 P_{\mu}^{\mu^{\prime}}+U_{\mu}^{\mu^{\prime}}, \\
Y_{2 \mu \nu}{ }^{\mu^{\prime} \nu^{\prime}} & \left.=U \delta_{\mu}{ }^{\left[\mu^{\prime}\right.} \delta_{\nu}^{\left.\nu^{\prime}\right]}+2\left(2 P_{[\mu}^{\left[\mu^{\prime}\right.}+U_{[\mu}^{\left[\mu^{\prime}\right.}\right) \delta_{\nu]} \nu^{\prime}\right]-W_{\mu \nu}{ }^{\mu^{\prime} \nu^{\prime},},
\end{aligned}
$$

where

$$
U=\frac{1}{2} \nabla_{\mu} v^{\mu}+\frac{1}{4} v_{\mu} v^{\mu}, \quad U_{\mu \nu}=U_{\nu \mu}=-\nabla_{\mu} v_{\nu}, \quad v_{\mu}=g^{2} \partial_{\mu} \frac{1}{g^{2}} .
$$

From (3.11) $\delta_{\sigma} W$ in (3.8) is then determined in the form (2.1) with

$$
L_{6}=\operatorname{tr}_{\Omega^{(2)}}\left(a_{\Delta^{(2)}, 3} \mid\right)-2 \operatorname{tr}_{\Omega^{(1)}}\left(a_{\Delta^{(1)}, 3} \mid\right)+3 \operatorname{tr}_{\Omega^{(0)}}\left(a_{\Delta^{(0)}, 3} \mid\right)+\nabla_{\mu} \nabla_{\nu} Z^{\mu \nu},
$$

up to the arbitrariness in $(2.3)$. Here $\operatorname{tr}_{\Omega^{(2)}}(1)=15, \operatorname{tr}_{\Omega^{(1)}}(1)=6, \operatorname{tr}_{\Omega^{(0)}}(1)=1$. The various traces necessary to determine (3.14) using (3.11) are given in appendix D. Neglecting the terms involving $U$ we get

$$
L_{6}^{R}=\frac{1}{7 !}\left(-\frac{1}{3} 8008 I_{1}-\frac{1}{3} 2378 I_{2}+180 I_{3}+442 E_{6}\right),
$$

which reproduces the results of [20] listed in (2.16). In terms of $v_{\mu}$ defined in (3.13)

$$
\begin{aligned}
L_{6}^{g}= & -\frac{1}{8} \partial^{\lambda} \nabla_{\mu} v^{\mu} \partial_{\lambda} \nabla_{\nu} v^{\nu}+2 P_{\mu \nu} \nabla^{\mu} v^{\nu} \nabla_{\lambda} v^{\lambda}-\frac{3}{4} \hat{R} \nabla_{\mu} v^{\mu} \nabla_{\nu} v^{\nu} \\
& -\left(B_{\mu \nu}+6 P_{\mu \lambda} P_{\nu}^{\lambda}-4 P_{\mu \nu} \hat{R}+\nabla_{\mu} \partial_{\nu} \hat{R}\right) v^{\mu} v^{\nu}+\left(P^{\lambda \rho} P_{\lambda \rho}-\hat{R}^{2}+\frac{1}{2} \nabla^{2} \hat{R}\right) v_{\mu} v^{\mu} \\
& +\frac{1}{3} W^{\mu \lambda \rho \omega} W_{\lambda \rho \omega}^{\nu} v_{\mu} v_{\nu}-\frac{11}{120} W^{\mu \nu \lambda \rho} W_{\mu \nu \lambda \rho} v^{\omega} v_{\omega} \\
& -\frac{1}{4}\left(\left(\nabla^{\mu} v^{\nu} \nabla_{\mu} v_{\nu}-\frac{1}{4} \nabla_{\mu} v^{\mu} \nabla_{\nu} v^{\nu}\right) v^{\rho} v_{\rho}+\hat{R}\left(v^{\rho} v_{\rho}\right)^{2}\right) \\
& -\frac{1}{32} v^{\mu} v_{\mu} \Delta_{2}\left(v^{\nu} v_{\nu}\right)-\frac{1}{64}\left(v^{\mu} v_{\mu}\right)^{3}
\end{aligned}
$$


for $\Delta_{2}=-\nabla^{2}+2 \hat{R}$. The first two lines in (3.16) agree with the form expected from $S_{1}+S_{2}+S_{3}$ given by (2.17), (2.20) and (2.24) and there are also contributions which may be identified with $j_{2}, j_{3}$ in $(2.26),(2.29)$, with coefficients $-\frac{1}{16},-\frac{1}{4}$, as well as $(2.32)$.

\section{Calculations in scalar $\phi^{3}$ theory}

In six dimensions the only conventionally renormalisable quantum field theory is the apparently unphysical (although for imaginary couplings the theory has relevance in statistical physics [25]) $\phi^{3}$ theory given by the Lagrangian

$$
\mathscr{L}(\phi, V)=-\frac{1}{2}\left(\partial^{\mu} \phi_{i} \partial_{\mu} \phi_{i}+\xi_{d} \phi_{i} \phi_{i} R\right)-V(\phi), \quad V(\phi)=\frac{1}{6} \lambda_{i j k} \phi_{i} \phi_{j} \phi_{k}, \quad i=1, \ldots, n_{\phi},
$$

where Weyl invariance in six dimensions requires $\xi_{6}=\frac{1}{5}$. However using dimensional regularisation with $d=6-\varepsilon$ it is necessary to keep $\varepsilon$-dependent terms to ensure compatibility with conformal constraints to two loop order so that $\xi_{d}=\frac{1}{5}-\frac{1}{100} \varepsilon+\mathrm{O}\left(\varepsilon^{2}\right)$. Two loop calculations for six dimensional $\phi^{3}$ theory on curved backgrounds were initiated in $[26,27]$ and recently extended to local couplings in [17] while the $\beta$-function has been determined to three loops in [28-32].

For a finite perturbative expansion starting from (4.1) it is necessary of course to add counterterms $\mathscr{L}_{\text {c.t. }}$ containing poles in $\varepsilon$. These may be restricted to the form, up to total derivatives,

$$
\mathscr{L}_{\text {c.t. }}(\phi, V) \equiv-\frac{1}{2} \operatorname{tr}\left(\partial^{\mu} \tilde{\varphi} N \partial_{\mu} \tilde{\varphi}+\xi_{d} \tilde{\varphi} N \tilde{\varphi} R\right)-V_{\text {c.t. }}(\tilde{\varphi}), \quad \tilde{\varphi}_{i j}=\lambda_{i j k} \phi_{k} .
$$

$V_{\text {c.t. }}(\tilde{\varphi})$ is a polynomial of degree three and includes $\phi$-independent terms of dimension six depending on the curvature and derivatives of the couplings. Renormalisability on a curved background and with local couplings dictates that in $(4.1) \mathscr{L}(\phi, V)$ should be extended to $\mathscr{L}(\phi, V, a)$ depending on a background gauge field $a_{\mu i j}=-a_{\mu j i}$ and also a general cubic $V$,

$$
\partial_{\mu} \phi_{i} \rightarrow\left(D_{\mu} \phi\right)_{i}=\partial_{\mu} \phi_{i}+a_{\mu i j} \phi_{j}, \quad V(\phi)=\frac{1}{6} \lambda_{i j k} \phi_{i} \phi_{j} \phi_{k}+\frac{1}{2} m_{i j} \phi_{i} \phi_{j}+h_{i} \phi_{i},
$$

so that

$$
\mathscr{L}_{0} \equiv \mathscr{L}(\phi, V, a)+\mathscr{L}_{\text {c.t. }}(\phi, V, a)=\mathscr{L}\left(\phi_{0}, V_{0}, a_{0}\right)-\frac{1}{(4 \pi)^{3}} \chi(V, a) .
$$

Here $\chi$ is a dimension six scalar independent of $\phi$ and formed from the curvature and the couplings with derivatives.

The RG equations take the form

$$
(4 \pi)^{\frac{1}{2} d}\left(\delta_{\sigma}+d \sigma+\mathcal{D}_{\beta}+\mathcal{D}_{\phi}\right) \mathscr{L}_{0}=\sigma L_{6}+\nabla_{\mu}\left(\mathcal{X}^{\mu \nu} \partial_{\nu} \sigma\right)
$$

for

$$
\begin{aligned}
& \mathcal{D}_{\beta}=\int \mathrm{d}^{d} x \sigma\left(\hat{\beta}_{\lambda i j k} \frac{\delta}{\delta \lambda_{i j k}}+\hat{\beta}_{m i j} \frac{\delta}{\delta m_{i j}}+\hat{\beta}_{h i} \frac{\delta}{\delta h_{i}}+\left(\rho \cdot D_{\mu} \lambda\right)_{i j} \frac{\delta}{\delta a_{\mu i j}}\right), \\
& \mathcal{D}_{\phi}=-\int \mathrm{d}^{d} x \sigma\left(\frac{1}{2}(d-2) \delta_{i j}+\gamma_{i j}\right) \phi_{j} \frac{\delta}{\delta \phi_{i}} .
\end{aligned}
$$


In (4.6) $\hat{\beta}_{\lambda i j k}=-\frac{1}{2} \varepsilon \lambda_{i j k}+\beta_{\lambda i j k}, \hat{\beta}_{m i j}=-2 m_{i j}+\left(\gamma_{m} \cdot m\right)_{i j}+\beta_{m i j}$, with $\beta_{m i j}$ independent of $m$, and $\hat{\beta}_{h i}=-\left(\frac{1}{2}(d+2) \delta_{i j}-\gamma_{i j}\right) h_{j}+\beta_{h i}$, with $\beta_{h}$ independent of $h$. $\mathcal{D}_{\beta}$ may contain additional terns involving $\partial_{\mu} \sigma$ but these are neglected as they are unimportant here. As usual (4.5) determines the higher order $\varepsilon$ poles in $\mathscr{L}_{0}$.

As shown by Brown and Collins in four dimensions for $\phi^{4}$ theory [33] the subtraction prescription implied by (4.2) suffices to ensure Weyl invariance remains valid to one loop order so that results at two loops for the $\phi$-independent counterterms should be consistent with the general constraints described here. At one loop the necessary counterterms are determined by $a_{\Delta, 3} \mid$ for the operator $\Delta=\left(-\nabla^{2}+\frac{1}{2}(d-2) \hat{R}\right) 1+m+\tilde{\varphi}$ which gives

$$
\begin{aligned}
(4 \pi)^{3} V_{\text {c.t. }}(\tilde{\varphi})^{(1)}=\frac{1}{\varepsilon}( & -\frac{1}{6} \operatorname{tr}\left((m+\tilde{\varphi})^{3}\right)-\frac{1}{180} \operatorname{tr}(m+\tilde{\varphi}) W^{\rho \mu \nu \lambda} W_{\rho \mu \nu \lambda} \\
& +\frac{1}{6} \operatorname{tr}\left(\tilde{\varphi}\left(\nabla^{2}-2 \hat{R}\right) m\right)-\frac{1}{12} \operatorname{tr}\left(\partial^{\mu} m \partial_{\mu} m+2 \hat{R} m^{2}\right) \\
& \left.+n_{\phi} \frac{1}{7 !}\left(\frac{5}{9} E_{6}-\frac{28}{3} I_{1}+\frac{5}{3} I_{2}+2 I_{3}\right)\right), \\
(4 \pi)^{3} N^{(1)}=- & \frac{1}{6 \varepsilon} .
\end{aligned}
$$

The one loop results for $\beta_{\lambda}, \gamma$ are standard, and are given in [17], but in addition we must take $\left(\rho^{(1)} \cdot \mathrm{d} \lambda\right)_{i j}=-\frac{1}{12}\left(\lambda_{i k l} \mathrm{~d} \lambda_{j k l}-\mathrm{d} \lambda_{i k l} \lambda_{j k l}\right), \beta^{(1)}{ }_{m i j}=-\frac{1}{6} \partial^{\mu} \lambda_{i k l} \partial_{\mu} \lambda_{j k l}$ as well as $\left.\beta^{(1)}{ }_{h i}\right|_{m=0}=-\frac{1}{180} \lambda_{i j j} W^{\rho \mu \nu \lambda} W_{\rho \mu \nu \lambda}$. For the scalar theory defined by (4.1) it is then easy to read off

$$
L_{6}^{R(1)}=n_{\phi} \frac{1}{7 !}\left(\frac{5}{9} E_{6}-\frac{28}{3} I_{1}+\frac{5}{3} I_{2}+2 I_{3}\right)
$$

which of course confirms the results for free scalar fields in (2.16).

Extending the calculations to two loops, letting $\lambda_{i j k} \rightarrow(4 \pi)^{\frac{3}{2}} \lambda_{i j k}$, leads to

$$
L_{6}^{R(2)}=\frac{\lambda_{i j k} \lambda_{i j k}}{9 \times 6 !}\left(\frac{2}{9} I_{1}-\frac{13}{18} I_{2}-\frac{1}{4} I_{3}\right)
$$

This is in agreement with similar two loop calculations in $[26,27]^{2}$ although a non conformal tensorial basis was used in these papers.

The two loop calculations may also be extended to allow for $x$-dependent couplings leading to contributions to $\chi^{(2)}$ in (4.4) involving derivatives of $\lambda$. There is a single double pole in $\varepsilon$, independent of $\phi$, which is proportional to $\partial^{\omega} \lambda_{i j k} \partial_{\omega} \lambda_{i j k} W^{\rho \mu \nu \lambda} W_{\rho \mu \nu \lambda}$ whose coefficient is in accord with (4.5), although it is necessary to take account of the $m$ terms in (4.7). Discarding terms with two overall derivatives and also some scheme dependent terms proportional to $W^{\rho \mu \nu \lambda} W_{\rho \mu \nu \lambda}$ these may be reduced to a conformally covariant form

\footnotetext{
${ }^{2}$ In [26] the relevant results are contained in (3.21) but it is necessary to have an additional factor $\varepsilon$ in the $R^{2}(\xi R+\ldots)$ term.
} 
and give, after rescaling $\lambda$ as before to absorb factors of $4 \pi$,

$$
\begin{aligned}
L_{6}^{g(2)}=-\frac{1}{6 \times 6 !}\left(\partial^{\mu} \nabla^{2} \lambda_{i j k} \partial_{\mu} \nabla^{2} \lambda_{i j k}-16 P^{\mu \nu} \nabla^{2} \lambda_{i j k} \nabla_{\mu} \partial_{\nu} \lambda_{i j k}+6 \hat{R} \nabla^{2} \lambda_{i j k} \nabla^{2} \lambda_{i j k}\right. \\
+8\left(B^{\mu \nu}+6 P^{\mu \lambda} P_{\lambda}^{\nu}-4 P^{\mu \nu} \hat{R}+\nabla^{\mu} \partial^{\nu} \hat{R}\right) \partial_{\mu} \lambda_{i j k} \partial_{\nu} \lambda_{i j k} \\
\left.\quad-4\left(2 P^{\lambda \rho} P_{\lambda \rho}-2 \hat{R}^{2}+\nabla^{2} \hat{R}\right) \partial^{\mu} \lambda_{i j k} \partial_{\mu} \lambda_{i j k}\right) \\
-\frac{4}{9 \times 6 !} W^{\mu \lambda \rho \omega} W_{\lambda \rho \omega}^{\nu} \partial_{\mu} \lambda_{i j k} \partial_{\nu} \lambda_{i j k}-\frac{23}{180 \times 6 !} W^{\nu \lambda \rho \omega} W_{\nu \lambda \rho \omega} \partial^{\mu} \lambda_{i j k} \partial_{\mu} \lambda_{i j k} .
\end{aligned}
$$

This has exactly the form expected from (2.25) and shows the presence of all three possible two index tensors on the conformal manifold although the coefficient of the last term in (4.10) is scheme dependent.

\section{Positivity constraints}

The various terms present in $L_{4}, L_{6}$ correspond to contact terms for identities resulting from Weyl scaling for correlation functions of the operators $\mathcal{O}_{I}$ coupled to the marginal couplings $g^{I}$ and also the energy momentum tensor. Positivity conditions arise most straightforwardly by considering two point functions. Restricting $\sigma$ to be a constant then (2.1) is equivalent to

$$
(4 \pi)^{\frac{d}{2}} \mu \frac{\partial}{\partial \mu} W=\int \mathrm{d}^{d} x \sqrt{\gamma} L_{d},
$$

for $\mu$ a regularisation scale and where, by analytic continuation, the metric is taken to be Euclidean and $i W \rightarrow W$. Applied to the two point function, obtained by functional differentiation of $W$ twice with respect to $g$, (5.1) requires

$$
\left.\mu \frac{\partial}{\partial \mu}\left\langle\mathcal{O}_{I}(x) \mathcal{O}_{J}(0)\right\rangle\right|_{\partial g=0, \gamma_{\mu \nu}=\delta_{\mu \nu}}= \begin{cases}g_{I J}\left(\partial^{2}\right)^{2} \delta^{4}(x) /(4 \pi)^{2}, & d=4, \\ g_{I J}\left(\partial^{2}\right)^{3} \delta^{6}(x) /(4 \pi)^{3}, & d=6 .\end{cases}
$$

Conformal invariance dictates

$$
\left.\left\langle\mathcal{O}_{I}(x) \mathcal{O}_{J}(0)\right\rangle\right|_{\partial g=0, \gamma_{\mu \nu}=\delta_{\mu \nu}}=G_{I J} \mathcal{R} \frac{1}{\left(x^{2}\right)^{d}} .
$$

For general $d,\left(x^{2}\right)^{-\alpha}$ may be defined as an analytic function in $\alpha$ with poles at $\alpha=$ $\frac{1}{2} d+n, n=0,1,2, \ldots$ Hence for $d$ even it is necessary to regularise, denoted in (5.3) by $\mathcal{R}$, so that $\left(x^{2}\right)^{-d}$ makes sense as a distribution for all $x$, or equivalently has a well defined Fourier transform. This is essential in order to make a connection with the identities in (5.2) and requires the introduction of the arbitrary scale $\mu$. A convenient prescription is provided by differential regularisation [34], which gives

$$
\begin{array}{ll}
\mathcal{R} \frac{1}{\left(x^{2}\right)^{4}}=-\frac{1}{4^{4} \times 3}\left(\partial^{2}\right)^{3}\left(\frac{1}{x^{2}} \ln \mu^{2} x^{2}\right), & d=4, \\
\mathcal{R} \frac{1}{\left(x^{2}\right)^{6}}=-\frac{1}{4^{6} \times 45}\left(\partial^{2}\right)^{4}\left(\frac{1}{\left(x^{2}\right)^{2}} \ln \mu^{2} x^{2}\right), & d=6 .
\end{array}
$$

Substituting (5.3) with (5.4) on the left hand side of (5.2) gives

$$
\left(2 \pi^{2}\right)^{2} G_{I J}=24 g_{I J}, \quad d=4, \quad \pi^{6} G_{I J}=360 g_{I J}, \quad d=6 .
$$


Unitarity implies positivity conditions on $G_{I J}$. To apply unitarity here it is sufficient to use the Fourier transforms

$$
\begin{aligned}
\int \mathrm{d}^{4} x e^{i k \cdot x} \frac{1}{x^{2}} \ln \mu^{2} x^{2} & =-\frac{4 \pi^{2}}{k^{2}} \ln \frac{e^{2 \gamma} k^{2}}{4 \mu^{2}}, \\
\int \mathrm{d}^{6} x e^{i k \cdot x} \frac{1}{\left(x^{2}\right)^{2}} \ln \mu^{2} x^{2} & =-\frac{4 \pi^{3}}{k^{2}}\left(\ln \frac{e^{2 \gamma} k^{2}}{4 \mu^{2}}-1\right),
\end{aligned}
$$

where $\gamma$ is the Euler-Mascheroni constant. It is then straightforward from (5.6) to determine the Fourier transforms of $\mathcal{R} \frac{1}{\left(x^{2}\right)^{4}}, \mathcal{R} \frac{1}{\left(x^{2}\right)^{6}}$ as given by (5.4) for $d=4,6$. Under analytic continuation from Euclidean to Minkowski space $k_{d} \rightarrow-i k_{0}$ and the absorptive part for $k^{2}<0$ is given by $\operatorname{Im} \ln \left(k^{2}-i \epsilon\right)=-\pi \theta\left(-k^{2}\right)$. Applied to (5.3) this requires positivity of $G_{I J}$.

For free scalar theories $(4 \pi)^{3}\left\langle\frac{1}{6} \phi^{3}(x) \frac{1}{6} \phi^{3}(0)\right\rangle=1 /\left(6 \pi^{6}\left(x^{2}\right)^{6}\right)$ so that in (5.3) we may take $G_{I J}=\delta_{I J} /\left(6 \pi^{6}\right)$. Using $(5.5) g_{I J}=\delta_{I J} /(3 \times 6$ !) in agreement with (4.10). For two-forms, from (3.1), (3.2),

$$
\begin{aligned}
\left\langle B^{\mu \nu}(x) B_{\lambda \rho}(0)\right\rangle & =\frac{g^{2}}{2 \pi^{3}} \delta_{\lambda}^{[\mu} \delta_{\rho}^{\nu]} \frac{1}{\left(x^{2}\right)^{2}}, \\
\left\langle(\mathrm{~d} B)^{\mu \nu \omega}(x) \mathrm{d} B_{\lambda \rho \sigma}(0)\right\rangle & =\frac{18 g^{2}}{\pi^{3}} I_{\lambda}^{[\mu}(x) I_{\rho}^{\nu}(x) I_{\sigma}^{\omega]}(x) \frac{1}{\left(x^{2}\right)^{3}},
\end{aligned}
$$

where

$$
I_{\mu \nu}(x)=\delta_{\mu \nu}-2 \frac{x_{\mu} x_{\nu}}{x^{2}}
$$

is the inversion tensor. In this case for $\mathcal{O}=\frac{1}{12}(\mathrm{~d} B)^{\mu \nu \omega}(\mathrm{d} B)_{\mu \nu \omega}$ then $\pi^{6} G_{I J} \rightarrow 90 g^{4}$ so that $g_{I J} \rightarrow \frac{1}{4} g^{4}$. This is in agreement with (3.16).

These considerations may also be applied to the energy momentum tensor defined by functional differentiation with respect to the metric. For the two point function only the Weyl anomaly proportional to $c$ in (2.8) contributes to the corresponding equation to (5.2) when $d=4$; for $d=6$ just the term $W^{\rho \mu \nu \lambda} \nabla^{2} W_{\rho \mu \nu \lambda}$, contained in $I_{3}$ and proportional to $c_{3}$, in (2.13) is relevant. Thus

$$
\left.\mu \frac{\partial}{\partial \mu}\left\langle T_{\mu \nu}(x) T_{\sigma \rho}(0)\right\rangle\right|_{\partial g=0, \gamma_{\mu \nu}=\delta_{\mu \nu}}= \begin{cases}4 c \mathcal{D}_{\mu \nu \sigma \rho} \delta^{4}(x) /(4 \pi)^{2}, & d=4, \\ 6 c_{3} \mathcal{D}_{\mu \nu \sigma \rho} \partial^{2} \delta^{6}(x) /(4 \pi)^{3}, & d=6,\end{cases}
$$

where, for general $d$,

$$
\mathcal{D}_{\mu \nu \sigma \rho}=\frac{1}{2}\left(S_{\mu \sigma} S_{\nu \rho}+S_{\mu \rho} S_{\nu \sigma}\right)-\frac{1}{d-1} S_{\mu \nu} S_{\sigma \rho}, \quad S_{\mu \nu}=\partial_{\mu} \partial_{\nu}-\delta_{\mu \nu} \partial^{2}
$$

For conformal theories

$$
\left.\left\langle T_{\mu \nu}(x) T_{\sigma \rho}(0)\right\rangle\right|_{\partial g=0, \gamma_{\mu \nu}=\delta_{\mu \nu}}=C_{T} \mathcal{R}\left(\frac{1}{\left(x^{2}\right)^{d}} \mathcal{I}_{\mu \nu \sigma \rho}(x)\right),
$$

with the inversion tensor for symmetric traceless rank two tensors

$$
\mathcal{I}_{\mu \nu \sigma \rho}=\frac{1}{2}\left(I_{\mu \sigma} I_{\nu \rho}+I_{\mu \rho} I_{\nu \sigma}\right)-\frac{1}{d} \delta_{\mu \nu} \delta_{\sigma \rho} .
$$


Since

$$
\mathcal{D}_{\mu \nu \sigma \rho} \frac{1}{\left(x^{2}\right)^{d-2}}=4(d-2)^{2} d(d+1) \frac{1}{\left(x^{2}\right)^{d}} \mathcal{I}_{\mu \nu \sigma \rho}(x),
$$

then in (5.11) we may define

$$
\begin{array}{ll}
\mathcal{R}\left(\frac{1}{\left(x^{2}\right)^{4}} \mathcal{I}_{\mu \nu \sigma \rho}(x)\right)=-\frac{1}{4^{4} \times 5} \mathcal{D}_{\mu \nu \sigma \rho} \partial^{2}\left(\frac{1}{x^{2}} \ln \mu^{2} x^{2}\right), & d=4, \\
\mathcal{R}\left(\frac{1}{\left(x^{2}\right)^{6}} \mathcal{I}_{\mu \nu \sigma \rho}(x)\right)=-\frac{1}{4^{6} \times 63} \mathcal{D}_{\mu \nu \sigma \rho}\left(\partial^{2}\right)^{2}\left(\frac{1}{\left(x^{2}\right)^{2}} \ln \mu^{2} x^{2}\right), & d=6 .
\end{array}
$$

Hence

$$
\left(2 \pi^{2}\right)^{2} C_{T}=160 c, \quad d=4, \quad \pi^{6} C_{T}=\frac{3}{5} \times 7 ! c_{3}, \quad d=6 .
$$

The relation between $C_{T}$ and $c$ for $d=4$ was obtained in [35] and the connection between $C_{T}$ and $c_{3}$ for $d=6$ in [20]. For $d=6$ the results in (2.16) are in agreement with calculations of $C_{T}$ for scalars, fermions in [35] and also two-form gauge fields in [36]. The results (5.6) suffice to determine the Fourier transforms of (5.14). Under continuation to Minkowski space we must take $T_{d i} \rightarrow-i T_{0 i}, i=1, \ldots, d-1, T_{d d} \rightarrow-T_{00}$, so that in (5.11) $\delta_{\mu \nu} \rightarrow \eta_{\mu \nu}$. It follows directly that unitarity requires $C_{T}>0$.

Positivity conditions for conserved vector currents $V_{a \mu}$ may be obtained in a similar fashion. Correlation functions containing $V_{a \mu}$ are defined by functional differentiation of $W$ with respect to a background gauge field $A_{a \mu}$. Then, from (2.8) and (2.15), taking $L_{6}^{F} \rightarrow-\frac{1}{4} \kappa_{a b} F_{a}^{\mu \nu} \nabla^{2} F_{b \mu \nu}$,

$$
\left.\mu \frac{\partial}{\partial \mu}\left\langle V_{a \mu}(x) V_{b \nu}(0)\right\rangle\right|_{\partial g=0, \gamma_{\mu \nu}=\delta_{\mu \nu}}= \begin{cases}-\kappa_{a b} S_{\mu \nu} \delta^{4}(x) /(4 \pi)^{2}, & d=4, \\ -\kappa_{a b} S_{\mu \nu} \partial^{2} \delta^{6}(x) /(4 \pi)^{3}, & d=6 .\end{cases}
$$

For conformal theories the vector two point function has the form

$$
\left.\left\langle V_{a \mu}(x) V_{b \nu}(0)\right\rangle\right|_{\partial g=0, \gamma_{\mu \nu}=\delta_{\mu \nu}}=C_{V a b} \mathcal{R}\left(\frac{1}{\left(x^{2}\right)^{d-1}} I_{\mu \nu}(x)\right) .
$$

In this case

$$
S_{\mu \nu} \frac{1}{\left(x^{2}\right)^{d-2}}=-2(d-2)(d-1) \frac{1}{\left(x^{2}\right)^{d-1}} I_{\mu \nu}(x),
$$

so that in (5.17) we may take

$$
\begin{array}{ll}
\mathcal{R}\left(\frac{1}{\left(x^{2}\right)^{3}} I_{\mu \nu}(x)\right)=\frac{1}{48} S_{\mu \nu} \partial^{2}\left(\frac{1}{x^{2}} \ln \mu^{2} x^{2}\right), & d=4, \\
\mathcal{R}\left(\frac{1}{\left(x^{2}\right)^{5}} I_{\mu \nu}(x)\right)=\frac{1}{3840} S_{\mu \nu}\left(\partial^{2}\right)^{2}\left(\frac{1}{\left(x^{2}\right)^{2}} \ln \mu^{2} x^{2}\right), & d=6 .
\end{array}
$$

Hence (5.16) requires

$$
\left(2 \pi^{2}\right)^{2} C_{V a b}=\frac{3}{2} \kappa_{a b}, \quad d=4, \quad \pi^{6} C_{V a b}=\frac{15}{2} \kappa_{a b}, \quad d=6 .
$$

The results for $\kappa$ in (2.16) agree with $C_{V}$ calculated for free scalars and fermions in [35]. 
There are further positivity constraints on the energy momentum tensor three point function which arise by requiring that the energy flux in light-like directions must be positive [21]. For $d=6$ the conditions take the form [37]

$$
\begin{aligned}
C_{1} & \equiv 1-\frac{1}{5} t_{2}-\frac{2}{35} t_{4} \geq 0, \quad C_{2} \equiv 1-\frac{1}{5} t_{2}-\frac{2}{35} t_{4}+\frac{1}{2} t_{2} \geq 0, \\
C_{3} & \equiv 1-\frac{1}{5} t_{2}-\frac{2}{35} t_{4}+\frac{4}{5}\left(t_{2}+t_{4}\right) \geq 0,
\end{aligned}
$$

with $t_{2}, t_{4}$ corresponding to the possible angular dependencies of the energy flux at null infinity. $t_{2}, t_{4}$ depend on the three possible structures for the conformal energy momentum tensor three point function after factoring $C_{T}$ as determining the overall normalisation. In six dimensions these are determined by the coefficients $c_{1}, c_{2}, c_{3}$ in the conformal anomaly (2.13) (unlike in four dimensions $a$ is irrelevant as far as the energy momentum tensor three point function is concerned). It is sufficient to use the results for free fields in [37] and (2.16) which give in general

$$
t_{2}=\frac{15\left(23 c_{1}-44 c_{2}+144 c_{3}\right)}{16 c_{3}}, \quad t_{4}=-\frac{105\left(c_{1}-2 c_{2}+6 c_{3}\right)}{2 c_{3}} .
$$

Then from (5.21) we may obtain, since $c_{3}>0$,

$$
\begin{aligned}
-21 c_{1}+36 c_{2}-128 c_{3} & \geq 0, \quad 101 c_{1}-196 c_{2}+\frac{1904}{3} c_{3} \geq 0, \\
-139 c_{1}+284 c_{2}-\frac{2432}{3} c_{3} & \geq 0 .
\end{aligned}
$$

The inequalities (5.21) define a triangular region in which the three free theory results correspond to the vertices where in each case two different inequalities become equalities.

For free scalars $C_{1}=C_{2}=0$. It is then non trivial that any conformal perturbation of a scalar theory should satisfy the inequalities (5.21). If we use the results for $c_{1}, c_{2}, c_{3}$ provided by (4.8) and (4.9) for $\phi^{3}$ theory with (5.22) we get

$$
C_{1}=\frac{7}{216} \lambda_{i j k} \lambda_{i j k}, \quad C_{2}=\frac{7}{36} \lambda_{i j k} \lambda_{i j k},
$$

so that the perturbative corrections respect the inequalities even though this theory remains potentially sick.

\section{Discussion}

The calculations in this paper show that there are significant differences between six and four dimensions and also two for which Zamolodchikov first derived the $c$-theorem. In two dimensions the result for the response to a Weyl rescaling in (2.1) becomes simply

$$
L_{2}=\frac{1}{6} c R-\frac{1}{2} g_{I J} \partial^{\mu} g^{I} \partial_{\mu} g^{J} .
$$

In this case the consistency conditions away from a conformal fixed point essentially imply

$$
\frac{1}{3} \partial_{I} c=g_{I J} \beta^{J}
$$


which implies irreversibility of RG flow, a strong version of the $c$-theorem, if $g_{I J}$ is positive definite. In this case positivity holds since $g_{I J}$ can be related directly to the two-point function for the operators $\mathcal{O}_{I}$ coupled to $g^{I}$. In four dimensions away from a fixed point there is no longer a single rank two tensor; in (2.8) the corresponding contributions become $\frac{1}{2} a_{I J} \nabla^{2} g^{I} \nabla^{2} g^{J}-G_{4}{ }^{\mu \nu} g_{I J} \partial_{\mu} g^{I} \partial_{\nu} g^{J}-\hat{R} f_{I J} \partial^{\mu} g^{I} \partial_{\mu} g^{J}$. In this case consistency conditions require

$$
\frac{1}{4} \partial_{I} a=g_{I J} \beta^{J}
$$

Only in the neighourhood of a conformal fixed point, when $a_{I J}=f_{I J}=g_{I J}$, does positivity of the two-point function, linked to $a_{I J}$, imply positivity of $g_{I J}$.

In six dimensions the results obtained in (2.25) show already that even at a conformal fixed point there are three two-index tensors. Away from a fixed point the RG flow equation becomes

$$
\frac{1}{12} \partial_{I} a=g_{1, I J} \beta^{J}
$$

involving $g_{1, I J}$, which away from the conformal point corresponds the contribution involving $G_{6}{ }^{\mu \nu} \sim W^{\mu \lambda \rho \omega} W^{\nu}{ }_{\lambda \rho \omega}$, rather than $g_{I J}$ which is related to the positive two point function. Hence, there are no straightforward positivity restrictions on $g_{1, I J}$ even near a fixed point. As shown by (4.10) $g_{1, I J}$ is negative for $\phi^{3}$ theory, which reproduces the challenge to a six dimensional $a$-theorem observed in [17]. In contrast, the calculations for the two-form case in (3.16) give a positive result $g_{1, I J}$. However, we should note that there is at present no argument implying that $a>0$ in six dimensions, unlike that given in [21] for the four dimensional $a$. In six dimensions $a$ is related to the energy momentum tensor four point function whose analysis is much harder than the three point function considered in [21]. Of course with supersymmetry there may be further relations between tensor structures which might link $g_{1, I J}, g_{2, I J}$ with $g_{I J}$.

In this paper we have focussed on solutions of the Weyl consistency conditions of the form given by (2.1), (2.2). Additional contributions to $\delta_{\sigma} W$ may be obtained by considering variations such that

$$
(4 \pi)^{\frac{d}{2}} \delta_{\sigma} W=\int \mathrm{d}^{d} x \sqrt{-\gamma} \partial_{\mu} \sigma Y_{d}^{\mu},
$$

where, if $Y_{d}^{\mu}$ is a total derivative, then it can generally be cancelled by local contributions to $W$. Alternative solutions of the consistency conditions may be obtained if $Y_{d}^{\mu}$ satisfies

$$
\begin{aligned}
\delta_{\sigma} Y_{d}^{\mu}+d \sigma Y_{d}^{\mu} & =\mathcal{Y}_{d}^{\mu \lambda \rho} \nabla_{\rho} \partial_{\lambda} \sigma+\nabla_{\rho}\left(\mathcal{Y}_{d}{ }^{\mu \lambda \rho} \partial_{\lambda} \sigma\right)+\mathcal{E}_{d}{ }^{\mu \lambda} \partial_{\lambda} \sigma \\
\mathcal{Y}_{d}{ }^{\mu \lambda \rho} & =-\mathcal{Y}_{d}{ }^{\lambda \mu \rho}, \quad \mathcal{E}_{d}{ }^{\mu \lambda}=\mathcal{E}_{d}{ }^{\lambda \mu}
\end{aligned}
$$

Of course contributions to $\mathcal{E}_{d}{ }^{\mu \lambda}$ of the form of $X_{d}{ }^{\mu \lambda}$ as in (2.2) may be discarded. In two and four dimensions examples are given by

$$
Y_{2}{ }^{\mu}=-w_{I} \partial^{\mu} g^{I}, \quad Y_{4}{ }^{\mu}=-2 G_{4}{ }^{\mu \nu} w_{I} \partial_{\nu} g^{I}+2 \partial_{[I} w_{J]} \partial^{\mu} g^{I} \nabla^{2} g^{J},
$$

where $w_{I} \mathrm{~d} g g^{I}$ is a one-form and in the four-dimensional case we make use of (A.16). In this case $\mathcal{Y}_{4}{ }^{\mu \lambda \rho}=2\left(\gamma^{\mu \rho} \gamma^{\lambda \nu}-\gamma^{\mu \nu} \gamma^{\lambda \rho}\right) w_{I} \partial_{\nu} g^{I}, \mathcal{E}_{4}{ }^{\mu \lambda}=0$. In (6.7) the normalisations have been chosen to agree with previous conventions. 
In six dimensions it is sufficient to take

$$
\begin{aligned}
Y_{6}{ }^{\mu}= & G_{6}{ }^{\mu \nu} w_{I} \partial_{\nu} g^{I} \\
+\partial_{[I} w_{J]} & \left(-\frac{10}{3} W^{\mu \lambda \rho \nu} \partial_{\nu} g^{I} \nabla_{\lambda} \partial_{\rho} g^{J}\right. \\
& +6 P^{\mu \nu} \partial^{\rho} g^{I} \nabla_{\nu} \partial_{\rho} g^{J}+6 P^{\rho \nu} \partial_{\rho} g^{I} \nabla^{\mu} \partial_{\nu} g^{J}-3 P^{\mu \nu} \partial_{\nu} g^{I} \nabla^{2} g^{J} \\
& \left.-6 \hat{R} \partial^{\nu} g^{I} \nabla^{\mu} \partial_{\nu} g^{J}-\frac{3}{2} \nabla^{\mu} \partial^{\nu} g^{I} \partial_{\nu} \nabla^{2} g^{J}+\frac{3}{4} \nabla^{2} g^{I} \partial^{\mu} \nabla^{2} g^{J}\right) \\
+ & \partial_{K} \partial_{[I} w_{J]}\left(\frac{1}{4} \nabla^{2} g^{K} \nabla^{2} g^{I} \partial^{\mu} g^{J}-\nabla^{\nu} \partial^{\rho} g^{K} \nabla_{\nu} \partial_{\rho} g^{I} \partial^{\mu} g^{J}-2 \nabla^{\mu} \partial^{\nu} g^{K} \nabla^{\rho} \partial_{\nu} g^{I} \partial_{\rho} g^{J}\right) .
\end{aligned}
$$

This satisfies (6.6) with

$$
\begin{aligned}
& \mathcal{Y}_{6}{ }^{\mu \lambda \rho}=-2 H_{6}{ }^{\mu \lambda \rho \nu} w_{I} \partial_{\nu} g^{I}+6 \partial_{[I} w_{J]} \gamma^{\rho[\mu} \nabla^{\lambda]} \partial^{\nu} g^{I} \partial_{\nu} g^{J}, \\
& \mathcal{E}_{6}{ }^{\mu \lambda}=-3 \partial_{[I} w_{J]}\left(\frac{1}{2} \gamma^{\mu \lambda} \partial^{\nu} \nabla^{2} g^{I} \partial_{\nu} g^{J}+\partial^{(\mu} \nabla^{2} g^{I} \partial^{\lambda)} g^{J}\right) \\
& +2 \partial_{K} \partial_{[I} w_{J]}\left(\gamma^{\mu \lambda} \partial^{\nu} g^{K} \nabla^{\rho} \partial_{\nu} g^{I} \partial_{\rho} g^{J}+\partial^{\rho} g^{K} \nabla^{(\mu} \partial_{\rho} g^{I} \partial^{\lambda)} g^{J}+3 \nabla^{(\mu} \partial^{\rho} g^{K} \partial_{\rho} g^{I} \partial^{\lambda)} g^{J}\right),
\end{aligned}
$$

where $H_{6}{ }^{\mu \lambda \rho \nu}$ is defined by (A.18) for $d=6$. We note that

$$
\begin{aligned}
Y_{6}^{\prime \mu}=w_{K I J}( & \frac{1}{4} \nabla^{2} g^{K} \nabla^{2} g^{I} \partial^{\mu} g^{J}-\nabla^{\nu} \partial^{\rho} g^{K} \nabla_{\nu} \partial_{\rho} g^{I} \partial^{\mu} g^{J} \\
& \left.+\nabla^{\mu} \partial^{\nu} g^{K} \nabla^{\rho} \partial_{\nu} g^{I} \partial_{\rho} g^{J}-2 \nabla^{\rho} \partial^{\nu} g^{K} \nabla^{\mu} \partial_{\nu} g^{I} \partial_{\rho} g^{J}\right)
\end{aligned}
$$

satisfies

$$
\delta_{\sigma} Y_{6}^{\prime \mu}+6 \sigma Y_{6}^{\prime \mu}=\mathcal{E}_{6}{ }^{\mu \lambda} \partial_{\lambda} \sigma
$$

for

$$
\begin{aligned}
\mathcal{E}_{6}^{\prime \mu \lambda}=w_{K I J} & \left(2 \partial^{\rho} g^{K} \nabla^{\mu} \partial^{\lambda} g^{I} \partial_{\rho} g^{J}-\gamma^{\mu \lambda} \partial^{\nu} g^{K} \nabla^{\rho} \partial \nu g^{I} \partial_{\rho} g^{J}\right. \\
& \left.+4 \partial^{\rho} g^{K} \nabla^{(\mu} \partial_{\rho} g^{I} \partial^{\lambda)} g^{J}-2 \partial^{(\mu} g^{K} \nabla^{\lambda)} \partial^{\rho} g^{I} \partial_{\rho} g^{J}\right),
\end{aligned}
$$

so long as $w_{K I J}=-w_{K J I}, w_{I J K}+w_{J K I}+w_{K I J}=0$. This gives rise to an ambiguity in the last line of (6.8) and correspondingly the last line of $\mathcal{E}_{6}{ }^{\mu \lambda}$ in (6.9). In (6.7) and (6.8) if $w_{I}=\partial_{I} u$ for any scalar $u$ defined on the conformal manifold then the variation (6.5) can be removed by a local contribution to $W$. To obtain a monotonic RG flow away from a critical point it is necessary to add a term linear in $w_{I} \beta^{I}$ to $c, a$ when $d=2,4$.

Despite the differences between six and two or four dimensions it is of course possible that further assumptions may lead to relations between the rank two tensors on the conformal manifold which could ensure that $g_{1, I J}$ is positive, at least in the neighbourhood of a fixed point, and that there is then a potential perturbative $a$-theorem. In particular this might be the case in supersymmetric theories but also when a non trivial six dimensional CFT has a holographic dual. In such cases there are arguments for an $a$-theorem which appear to be valid in any dimension [38-40]. Such arguments depend on positivity conditions for the bulk energy momentum tensor which are doubtless vitiated in any correspondence for $\phi^{3}$ theories. In any event, simple holographic duals may not be sensitive to the additional 
two index tensors revealed by our general discussion in six dimensions. Other arguments for a $c$, or $a$, theorem in six dimensions are given in [41]. This relates the variation of the free energy on a sphere as the radius varies to the metric defined by the two point function. A rather similar argument, restricted to four dimensions, is given in [42]. The relation to our analysis is not clear but the calculation is quite sensitive to the details of regularisation.

\section{Acknowledgments}

We are both grateful for the warm hospitality of KITP Santa Barbara where this work was initiated. We have benefitted from the Mathematica package xAct. HO would like to thank Adam Schwimmer at KITP and Ian Jack for many helpful discussions. The research of AS is supported in part by the National Science Foundation under Grant No. 1350180.

\section{A Conformal tensors, invariants and operators}

The anomalous terms in Weyl scaling identities are, for type B [19], expressed in terms of conformal scalars. These are in turn formed from conformal tensors which transform homogeneously, without any derivatives of $\sigma$. Concise expressions for these may be obtained by first defining a modified scalar curvature and the Schouten tensor

$$
\hat{R}=\frac{1}{2(d-1)} R, \quad P_{\mu \nu}=\frac{1}{d-2}\left(R_{\mu \nu}-\gamma_{\mu \nu} \hat{R}\right), \quad \gamma^{\mu \nu} P_{\mu \nu}=\hat{R}, \quad \nabla^{\nu} P_{\mu \nu}=\partial_{\mu} \hat{R},
$$

which have the crucial properties

$$
\delta_{\sigma} \hat{R}=-2 \sigma \hat{R}-\nabla^{2} \sigma, \quad \delta_{\sigma} P_{\mu \nu}=-\nabla_{\mu} \partial_{\nu} \sigma
$$

The Weyl tensor is then given in terms of the Riemann tensor by

$$
W_{\lambda \rho \mu \nu}=R_{\lambda \rho \mu \nu}-\gamma_{\lambda \mu} P_{\rho \nu}+\gamma_{\rho \mu} P_{\lambda \nu}+\gamma_{\lambda \nu} P_{\rho \mu}-\gamma_{\rho \nu} P_{\lambda \mu}
$$

To discuss tensors which transform homogeneously under Weyl rescaling it is necessary to consider the Cotton tensor defined by

$$
C_{\mu \nu \lambda}=\nabla_{\lambda} P_{\mu \nu}-\nabla_{\nu} P_{\mu \lambda},
$$

and also the Bach tensor given by

$$
\begin{aligned}
B_{\mu \nu} & =\nabla^{\lambda} C_{\mu \nu \lambda}-P^{\lambda \omega} W_{\lambda \mu \nu \omega} \\
& =-2 P^{\lambda \omega} W_{\lambda \mu \nu \omega}-d P_{\mu \lambda} P^{\lambda}{ }_{\nu}+\gamma_{\mu \nu} P_{\rho \lambda} P^{\rho \lambda}+\nabla^{2} P_{\mu \nu}-\nabla_{\mu} \nabla_{\nu} \hat{R} .
\end{aligned}
$$

These have the properties

$$
\begin{aligned}
C_{\mu \nu \lambda} & =-C_{\mu \lambda \nu}, \quad C_{\mu \nu \lambda}+C_{\lambda \mu \nu}+C_{\nu \lambda \mu}=0, \quad \gamma^{\mu \nu} C_{\mu \nu \lambda}=0, \quad \nabla^{\mu} C_{\mu \nu \lambda}=0, \\
B_{\mu \nu} & =B_{\nu \mu}, \quad \gamma^{\mu \nu} B_{\mu \nu}=0, \quad \nabla^{\nu} B_{\mu \nu}=(d-4) P^{\lambda \rho} C_{\lambda \rho \mu} .
\end{aligned}
$$

The Bianchi identity for the Weyl tensor becomes

$$
\begin{aligned}
& \nabla_{\omega} W_{\lambda \rho \mu \nu}+\nabla_{\mu} W_{\lambda \rho \nu \omega}+\nabla_{\nu} W_{\lambda \rho \omega \mu} \\
& =\gamma_{\lambda \mu} C_{\rho \omega \nu}+\gamma_{\rho \mu} C_{\lambda \nu \omega}+\gamma_{\lambda \nu} C_{\rho \mu \omega}+\gamma_{\rho \nu} C_{\lambda \omega \mu}+\gamma_{\lambda \omega} C_{\rho \nu \mu}+\gamma_{\rho \omega} C_{\lambda \mu \nu},
\end{aligned}
$$


from which $\nabla^{\rho} W_{\rho \mu \nu \lambda}=-(d-3) C_{\mu \nu \lambda}$. Under Weyl scalings $\delta_{\sigma} W_{\lambda \rho \mu \nu}=2 \sigma W_{\lambda \rho \mu \nu}$ and

$$
\delta_{\sigma} C_{\mu \nu \lambda}=-\partial^{\rho} \sigma W_{\rho \mu \nu \lambda}, \quad \delta_{\sigma} B_{\mu \nu}=-2 \sigma B_{\mu \nu}+(d-4) \partial^{\lambda} \sigma\left(C_{\mu \nu \lambda}+C_{\nu \mu \lambda}\right) .
$$

Since the Weyl tensor vanishes when $d=3$ the Cotton tensor is then a conformal tensor, as is the Bach tensor when $d=4$.

In terms of these expressions

$$
E_{4}=6 R_{\lambda \rho}{ }^{[\lambda \rho} R_{\mu \nu}{ }^{\mu \nu]}=W_{\lambda \rho}{ }^{\mu \nu} W_{\mu \nu}{ }^{\lambda \rho}-4(d-2)(d-3)\left(P^{\mu \nu} P_{\mu \nu}-\hat{R}^{2}\right),
$$

which is the Euler density in four dimensions, and also

$$
\begin{aligned}
E_{6}= & \left.90 R_{\lambda \rho}{ }^{[\lambda \rho} R_{\mu \nu}{ }^{\mu \nu} R_{\omega \tau}{ }^{\omega \tau]}\right] \\
= & 8 I_{1}+4 I_{2}+6(d-5)\left(\hat{R} W_{\lambda \rho}{ }^{\mu \nu} W_{\mu \nu}{ }^{\lambda \rho}-4 P_{\mu \nu} W^{\mu \lambda \rho \omega} W^{\nu}{ }_{\lambda \rho}\right) \\
& -24(d-4)(d-5) P_{\mu \nu} P_{\lambda \rho} W^{\mu \lambda \rho \nu} \\
& +8(d-3)(d-4)(d-5)\left(\hat{R}^{3}-3 \hat{R} P^{\mu \nu} P_{\mu \nu}+2 P^{\mu \nu} P_{\nu \lambda} P_{\mu}^{\lambda}\right),
\end{aligned}
$$

for $I_{1}, I_{2}$ conformal scalars

$$
I_{1}=W_{\rho \mu \nu \lambda} W^{\mu \omega \tau \nu} W_{\omega}^{\rho \lambda} \tau, \quad I_{2}=W_{\mu \nu}^{\lambda \rho} W_{\lambda \rho}{ }^{\omega \tau} W_{\omega \tau}^{\mu \nu} .
$$

These satisfy

$$
\begin{aligned}
& \delta_{\sigma} E_{4}+4 \sigma E_{4}=8(d-3) \nabla_{\mu}\left(G_{4}{ }^{\mu \nu} \partial_{\nu} \sigma\right), \\
& \delta_{\sigma} E_{6}+6 \sigma E_{6}=24(d-5) \nabla_{\mu}\left(G_{6}{ }^{\mu \nu} \partial_{\nu} \sigma\right)
\end{aligned}
$$

for

$$
G_{4}{ }^{\mu \nu}=(d-2)\left(P^{\mu \nu}-\gamma^{\mu \nu} \hat{R}\right)=R^{\mu \nu}-\frac{1}{2} \gamma^{\mu \nu} R,
$$

the Einstein tensor, and

$$
\begin{aligned}
G_{6}{ }^{\mu \nu}= & W^{\mu \lambda \rho \omega} W_{\lambda \rho \omega}^{\nu}+2(d-4) W^{\mu \lambda \rho \nu} P_{\lambda \rho}-2(d-3)(d-4)\left(P^{\mu \lambda} P_{\lambda}^{\nu}-P^{\mu \nu} \hat{R}\right) \\
& -\frac{1}{4} \gamma^{\mu \nu}\left(W^{\tau \lambda \rho \omega} W_{\tau \lambda \rho \omega}-4(d-3)(d-4)\left(P^{\lambda \rho} P_{\lambda \rho}-\hat{R}^{2}\right)\right),
\end{aligned}
$$

where $\nabla_{\mu} G_{4}{ }^{\mu \nu}=\nabla_{\mu} G_{6}{ }^{\mu \nu}=0$ and $G_{6}{ }^{\mu \nu}=0$ for $d=3,4$. For completeness we note that

$$
E_{2}=R, \quad \delta_{\sigma} E_{2}+2 \sigma E_{2}=2(d-1) \nabla_{\mu}\left(G_{2}{ }^{\mu \nu} \partial_{\nu} \sigma\right), \quad \text { for } \quad G_{2}{ }^{\mu \nu}=-\gamma^{\mu \nu} .
$$

It is useful to note that

$$
\delta_{\sigma} G_{4}{ }^{\mu \nu}+4 \sigma G_{4}{ }^{\mu \nu}=-(d-2) H_{4}{ }^{\mu \lambda \rho \nu} \nabla_{\lambda} \partial_{\rho} \sigma, \quad H_{4}{ }^{\mu \lambda \rho \nu}=\gamma^{\mu \rho} \gamma^{\lambda \nu}-\gamma^{\lambda \rho} \gamma^{\mu \nu},
$$

and

$$
\delta_{\sigma} G_{6}{ }^{\mu \nu}+6 \sigma G_{6}{ }^{\mu \nu}=-2(d-4) H_{6}{ }^{\mu \lambda \rho \nu} \nabla_{\lambda} \partial_{\rho} \sigma,
$$

for

$$
\begin{aligned}
H_{6}{ }^{\mu \lambda \rho \nu}= & W^{\mu \lambda \rho \nu} \\
& -(d-3)\left(\gamma^{\mu \rho} P^{\lambda \nu}-\gamma^{\lambda \rho} P^{\mu \nu}-\gamma^{\mu \nu} P^{\lambda \rho}+\gamma^{\lambda \nu} P^{\mu \rho}-\hat{R}\left(\gamma^{\mu \rho} \gamma^{\lambda \nu}-\gamma^{\lambda \rho} \gamma^{\mu \nu}\right)\right),
\end{aligned}
$$

where $\nabla_{\rho} H_{6}{ }^{\mu \lambda \rho \nu}=0, \gamma_{\lambda \rho} H_{6}{ }^{\mu \lambda \rho \nu}=(d-3) G_{4}{ }^{\mu \nu}$. 
Besides $I_{1}, I_{2}$ in (A.11) there is an additional conformal scalar of dimension six. For general $d$ it may be succinctly expressed as

$$
\begin{aligned}
\Omega= & \frac{1}{4}(10-d)\left(W^{\rho \mu \nu \lambda} \nabla^{2} W_{\rho \mu \nu \lambda}+4(d-2) C^{\mu \nu \lambda} C_{\mu \nu \lambda}\right) \\
& +\left(\frac{1}{8}(d-2) \nabla^{2}-4 \hat{R}\right) W^{\rho \mu \nu \lambda} W_{\rho \mu \nu \lambda} .
\end{aligned}
$$

Alternative forms $[22,43,44]$, equivalent to (A.22) up to contributions linear in $I_{1}, I_{2}$, can be obtained with the aid of the relations from (A.7)

$$
\begin{gathered}
4 I_{1}-I_{2}=W^{\rho \mu \nu \lambda} \nabla^{2} W_{\rho \mu \nu \lambda}-2(d-2) P_{\rho \omega} W^{\rho \mu \nu \lambda} W^{\omega}{ }_{\mu \nu \lambda}-2 \hat{R} W^{\rho \mu \nu \lambda} W_{\rho \mu \nu \lambda} \\
+2(d-2)(d-3) C^{\mu \nu \lambda} C_{\mu \nu \lambda}+2(d-2) \nabla_{\omega}\left(W^{\omega \mu \nu \lambda} C_{\mu \nu \lambda}\right), \\
(d-4) \nabla_{\omega}\left(W^{\omega \mu \nu \lambda} C_{\mu \nu \lambda}\right)=-\nabla_{\mu} \nabla_{\nu}\left(W^{\mu \lambda \rho \omega} W_{\lambda \rho \omega}^{\nu}\right)+\frac{1}{4} \nabla^{2}\left(W^{\mu \lambda \rho \omega} W_{\mu \lambda \rho \omega}\right) .
\end{gathered}
$$

The form used in [20] is given by

$$
I_{3}=(d-3) \Omega-\frac{1}{2}(10-d)\left(4 I_{1}-I_{2}\right),
$$

so that, for $d=6$,

$$
\begin{aligned}
I_{3}= & W^{\rho \mu \nu \lambda} \nabla^{2} W_{\rho \mu \nu \lambda}+16 P_{\mu \nu} W^{\mu \rho \lambda \omega} W_{\rho \lambda \omega}^{\nu}-8 \hat{R} W^{\rho \mu \nu \lambda} W_{\rho \mu \nu \lambda} \\
& +8 \nabla_{\mu} \nabla_{\nu}\left(W^{\mu \lambda \rho \omega} W_{\lambda \rho \omega}^{\nu}\right)-\frac{1}{2} \nabla^{2}\left(W^{\rho \mu \nu \lambda} W_{\rho \mu \nu \lambda}\right) .
\end{aligned}
$$

The $I_{r}$ all satisfy

$$
\delta_{\sigma} I_{r}+6 \sigma I_{r}=0 .
$$

Besides (A.20) we may also note the derivative relation

$$
\begin{aligned}
\nabla_{\mu} \nabla_{\nu}\left(P^{\mu \lambda} P_{\lambda}^{\nu}-2 P^{\mu \nu} \hat{R}\right)+\nabla^{2} \hat{R}^{2}= & P_{\mu \nu} P_{\lambda \rho} W^{\mu \lambda \rho \nu}+d P^{\mu \nu} P_{\nu \lambda} P_{\mu}^{\lambda}-\hat{R} P^{\mu \nu} P_{\mu \nu} \\
& -\frac{1}{2} C^{\lambda \mu \nu} C_{\lambda \mu \nu}+\nabla^{\lambda} P^{\mu \nu} \nabla_{\lambda} P_{\mu \nu}-\partial^{\lambda} \hat{R} \partial_{\lambda} \hat{R} .
\end{aligned}
$$

If a connection $A_{\mu}$, with corresponding field strength $F_{\mu \nu}$, is present, then there are further conformal scalars. Analogous to (A.19) there is a similar dimension six conformal scalar formed from $F_{\mu \nu}$ which as given in [43] has the form

$$
\begin{aligned}
\hat{\Omega}= & \frac{1}{4}(10-d)\left(\frac{1}{4}(d-4)\left(F^{\mu \nu} \mathcal{D}^{2} F_{\mu \nu}+\mathcal{D}^{2} F^{\mu \nu} F_{\mu \nu}\right)+\mathcal{D}_{\mu} F^{\mu \lambda} \mathcal{D}^{\nu} F_{\nu \lambda}\right) \\
& +\frac{1}{16}(d-4)\left((d-4) \mathcal{D}^{2}-24 \hat{R}\right) F^{\mu \nu} F_{\mu \nu},
\end{aligned}
$$

for $\mathcal{D}_{\mu}$ the appropriate covariant derivative, $\mathcal{D}_{\lambda} F_{\mu \nu}=\partial_{\lambda} F_{\mu \nu}+\left[A_{\lambda}, F_{\mu \nu}\right]$. Corresponding to (A.20), using the Bianchi identity for $F_{\mu \nu}$,

$$
\begin{aligned}
\mathcal{D}_{\mu} \mathcal{D}_{\nu}\left(F^{\mu \lambda} F_{\lambda}^{\nu}\right)-\frac{1}{2} \mathcal{D}^{2}\left(F^{\mu \nu} F_{\mu \nu}\right) \\
=\mathcal{D}_{\mu} F^{\mu \lambda} \mathcal{D}^{\nu} F_{\nu \lambda}-\frac{1}{2} \mathcal{D}^{\lambda} F^{\mu \nu} \mathcal{D}_{\lambda} F_{\mu \nu}-(d-4) P_{\mu \nu} F^{\mu \lambda} F_{\lambda}^{\nu}-\hat{R} F^{\mu \nu} F_{\mu \nu} \\
\quad+\frac{1}{2} W_{\mu \nu \lambda \rho} F^{\mu \nu} F^{\lambda \rho}-2 F^{\mu \nu} F_{\nu \lambda} F_{\mu}^{\lambda} .
\end{aligned}
$$


The terms in the last line are conformal scalars. Using (A.26) an expression similar to (A.22) can be obtained which is more convenient for our purposes. For $d=6$ this becomes

$$
\hat{I}=\frac{1}{2}\left(F^{\mu \nu} \mathcal{D}^{2} F_{\mu \nu}+\mathcal{D}^{2} F^{\mu \nu} F_{\mu \nu}\right)-4 \hat{R} F^{\mu \nu} F_{\mu \nu}+\left(2 \mathcal{D}_{\mu} \mathcal{D}_{\nu}+4 P_{\mu \nu}\right)\left(F^{\mu \lambda} F_{\lambda}^{\nu}\right),
$$

which corresponds to the form given in (2.15).

In addition to conformal tensors there are also conformally covariant differential operators $^{3}$ which play a crucial role. The conformal Laplacian, or Yamabe operator,

$$
\Delta_{2}=-\nabla^{2}+\frac{1}{2}(d-2) \hat{R}
$$

acts on scalars of dimension $\frac{1}{2}(d-2), \delta_{\sigma} \Delta_{2}=\frac{1}{2}(d-2) \Delta_{2} \sigma-\frac{1}{2}(d+2) \sigma \Delta_{2}$. For $d=10$ the conformal scalar $\Omega$ in (A.19) is just $-\Delta_{2} W^{\rho \mu \nu \lambda} W_{\rho \mu \nu \lambda}$. The corresponding fourth order Paneitz operator [45] was for $d=4$ found first by Fradkin and Tseytlin [46-48] and also rederived by Riegert [49],

$$
\Delta_{4}=\nabla^{2} \nabla^{2}+\nabla^{\mu}\left(4 P_{\mu \nu}-2 \gamma_{\mu \nu} \hat{R}\right) \partial^{\nu},
$$

acting on dimensionless scalars such that $\delta_{\sigma} \Delta_{4}=-4 \sigma \Delta_{4}$. This expression is equivalent to the result for $L_{4}$ in (2.8). There is a corresponding extension in six dimensions, given by Branson [50], which can be written as

$$
\begin{aligned}
\Delta_{6}= & -\nabla^{2} \nabla^{2} \nabla^{2}-8 \nabla^{2} P_{\mu \nu} \nabla^{\mu} \partial^{\nu}-8 \nabla^{\mu} \nabla^{\nu} P_{\mu \nu} \nabla^{2}+6 \nabla^{2} \hat{R} \nabla^{2} \\
& -\nabla^{\mu}\left(8 B_{\mu \nu}+8 \nabla_{\mu} \nabla_{\nu} \hat{R}+48 P_{\mu \lambda} P_{\nu}{ }^{\lambda}-32 P_{\mu \nu} \hat{R}\right) \partial^{\nu} \\
& +\nabla^{\mu}\left(8 P_{\rho \lambda} P^{\rho \lambda}-8 \hat{R}^{2}+4 \nabla^{2} \hat{R}\right) \partial_{\mu},
\end{aligned}
$$

so that $\delta_{\sigma} \Delta_{6}=-6 \sigma \Delta_{6}$. This operator is equivalent to the contributions $S_{1}+S_{2}+S_{3}$ as given by (2.17), (2.20), (2.24).

Besides acting on scalars there are also conformal differential operators for tensors with various symmetries. For our purposes we need only consider operators acting on symmetric traceless tensors of rank two. Adapting results from $[52,53]$ to this special case

$$
\begin{aligned}
\Delta_{2, T} h_{\mu \nu}= & \Delta_{2} h_{\mu \nu}+\frac{8}{d+2} \nabla_{(\mu} \nabla^{\lambda} h_{\nu) \lambda}+4 P_{(\mu}^{\lambda} h_{\nu) \lambda} \\
& -\frac{1}{d} \gamma_{\mu \nu}\left(\frac{8}{d+2} \nabla^{\rho} \nabla^{\lambda} h_{\rho \lambda}+P^{\rho \lambda} h_{\lambda \rho}\right)
\end{aligned}
$$

so that $\delta_{\sigma} \Delta_{2, T}=\frac{1}{2}(d-6) \Delta_{2, T} \sigma-\frac{1}{2}(d-2) \sigma \Delta_{2, T}$. The operators $\Delta_{2}$ and $\Delta_{2, T}$ are implicitly determined by the $j_{2}, j_{1}$ contributions in $T_{1}+T_{2}$ given by (2.26), (2.29).

The calculations for $\phi^{3}$ theory are based on using the heat kernel expansion for $e^{-t \Delta}$, with $\Delta=-\mathcal{D}^{2}+\frac{1}{2}(d-2) \hat{R}+Y$ in terms of the Seeley-DeWitt coefficients $a_{n}(x, y) . \Delta$ is a

\footnotetext{
${ }^{3}$ An overview and some useful expressions can be found in [51].
} 
conformal differential operator if we assume $\delta_{\sigma} Y=-2 \sigma Y$. If $A_{\mu}, Y=0$ then $\Delta=\Delta_{2}$ as in (A.28). For the diagonal coefficients $a_{n} \mid$, when $y=x$, we have

$$
\begin{aligned}
180 a_{2} \mid= & W^{\lambda \rho \mu \nu} W_{\lambda \rho \mu \nu}+15 F^{\mu \nu} F_{\mu \nu}+60 Y^{2}+30 \Delta Y \\
& -(d-6)\left((d-2) P^{\mu \nu} P_{\mu \nu}-\frac{1}{2}(5 d-16) \hat{R}^{2}+3 \nabla^{2} \hat{R}-15 \hat{R} Y\right) \\
= & \frac{3}{2} W^{\lambda \rho \mu \nu} W_{\lambda \rho \mu \nu}-\frac{1}{2} E_{4}+15 F^{\mu \nu} F_{\mu \nu}+90 Y^{2}+6 \nabla^{2} \hat{R}-30 \mathcal{D}^{2} Y \quad \text { if } \quad d=4,
\end{aligned}
$$

and from [54] for $A_{\mu}, Y=0$,

$$
\begin{aligned}
7 ! a_{3} \mid=-\frac{80}{9} I_{1}+ & \frac{44}{9} I_{2}+6 \Omega \\
+(d-8)( & -\frac{3}{2} \nabla^{\omega} W^{\rho \mu \nu \lambda} \nabla_{\omega} W_{\rho \mu \nu \lambda}-\frac{16}{3} P_{\rho \omega} W^{\rho \mu \nu \lambda} W^{\omega}{ }_{\mu \nu \lambda}-\frac{14}{3} \hat{R} W^{\rho \mu \nu \lambda} W_{\rho \mu \nu \lambda} \\
& +\frac{8}{3}(d+2) P_{\mu \nu} P_{\lambda \rho} W^{\mu \lambda \rho \nu}+8(d-2) C^{\mu \nu \lambda} C_{\mu \nu \lambda} \\
& +(d-2)\left(2 \nabla^{\lambda} P^{\mu \nu} \nabla_{\lambda} P_{\mu \nu}-4 \nabla^{2}\left(P^{\mu \nu} P_{\mu \nu}\right)-4 \nabla_{\mu} \nabla_{\nu}\left(P^{\mu \nu} \hat{R}\right)\right) \\
& -(5 d-22) \partial^{\lambda} \hat{R} \partial_{\lambda} \hat{R}+(9 d-32) \nabla^{2}\left(\hat{R}^{2}\right)-6 \nabla^{2} \nabla^{2} \hat{R} \\
& +\frac{8}{9}\left(d^{2}-4 d+12\right) P^{\mu \nu} P_{\nu \lambda} P^{\lambda}{ }_{\mu}+\frac{2}{3}\left(7 d^{2}-40 d+36\right) \hat{R} P^{\mu \nu} P_{\mu \nu} \\
& \left.-\frac{1}{9}\left(35 d^{2}-266 d+456\right) \hat{R}^{3}\right) .
\end{aligned}
$$

For $d=6$

$$
\begin{aligned}
7 ! a_{3} \mid= & \frac{5}{9} E_{6}-\frac{28}{3} I_{1}+\frac{5}{3} I_{2}+2 I_{3}+14\left(3 \hat{I}+5 W_{\mu \nu \lambda \rho} F^{\mu \nu} F^{\lambda \rho}-8 F^{\mu \nu} F_{\nu \lambda} F_{\mu}^{\lambda}\right) \\
& -7 !\left(\frac{1}{12} Y^{3}+\frac{1}{12} Y \Delta Y+\frac{1}{180} W^{\rho \mu \nu \lambda} W_{\rho \mu \nu \lambda} Y\right) \\
& -7 !\left(\frac{1}{30}\left(F^{\mu \nu} F_{\mu \nu} Y+Y F^{\mu \nu} F_{\mu \nu}\right)+\frac{1}{60} F^{\mu \nu} Y F_{\mu \nu}\right) \\
& -\nabla_{\mu} \nabla_{\nu}\left(12 W^{\mu \lambda \rho \omega} W_{\lambda \rho \omega}^{\nu}+16 P^{\mu \lambda} P_{\lambda}^{\nu}-64 P^{\mu \nu} \hat{R}\right) \\
& +\nabla^{2}\left(\frac{9}{2} W^{\lambda \rho \mu \nu} W_{\lambda \rho \mu \nu}+32 P^{\mu \nu} P_{\mu \nu}-60 \hat{R}^{2}\right)+12 \nabla^{2} \nabla^{2} \hat{R} \\
& -56 \mathcal{D}_{\mu} \mathcal{D}_{\nu}\left(F^{\mu \lambda} F_{\lambda}^{\nu}\right)+49 \mathcal{D}^{2}\left(F^{\mu \nu} F_{\mu \nu}\right) \\
& -7 !\left(\frac{1}{90} \mathcal{D}_{\mu} \mathcal{D}_{\nu}\left(G_{4}{ }^{\mu \nu} Y\right)-\frac{1}{24} \mathcal{D}^{2} Y^{2}+\frac{1}{60} \mathcal{D}^{2} \mathcal{D}^{2} Y\right)
\end{aligned}
$$

This gives the results in (3.11) and (4.7). The results in (A.32), (A.33) and (A.34) reflect the theorems of Parker and Rosenberg [43] $]^{4}$ that $a_{n} \mid$ for $d=2 n+2$ is a conformal scalar and for $d=2 n, \int \mathrm{d}^{2 n} x \sqrt{-\gamma} a_{n} \mid$ is a conformal invariant, and suggest the slight extension, that for $d=2 n, a_{n} \mid$ is a linear combination of conformal scalars and the Euler density $E_{2 n}$ up to terms with two derivatives.

\footnotetext{
${ }^{4}$ As noted in [20] their results contain some errors which are hopefully corrected in (A.33), (A.34).
} 


\section{B Expansion of six dimensional dilaton action}

In six dimensions $\mathcal{L}_{6}(\sigma)$ in (2.5) may be obtained by using (2.6). Starting from (2.14) we may straightforwardly use (A.2) successively in (2.6) to determine $X_{6, r}^{R \mu \nu}$ for $r=1,2,3,4$ and hence obtain

$$
\begin{aligned}
\mathcal{L}_{6}^{R}(\sigma)= & \sigma L_{6}^{R}-12 a G_{6}{ }^{\mu \nu} \partial_{\mu} \sigma \partial_{\nu} \sigma \\
+ & 16 a\left(W^{\mu \lambda \rho \nu} \nabla_{\lambda} \partial_{\rho} \sigma \partial_{\mu} \sigma \partial_{\nu} \sigma-6 P^{\mu \nu} \nabla^{\lambda} \partial_{\mu} \sigma \partial_{\nu} \sigma \partial_{\lambda} \sigma\right. \\
& +3 P^{\mu \nu} \nabla^{2} \sigma \nabla_{\mu} \partial_{\nu} \sigma+3 P^{\mu \nu} \nabla_{\mu} \partial_{\nu} \sigma \partial^{\lambda} \sigma \partial_{\lambda} \sigma \partial_{\lambda} \sigma \\
& \left.+3 \hat{R} \nabla^{\mu} \partial^{\nu} \sigma \partial_{\mu} \sigma \partial_{\nu} \sigma-3 \hat{R} \nabla^{2} \sigma \partial^{\lambda} \sigma \partial_{\lambda} \sigma\right) \\
& -24 a\left(\frac{5}{2} \hat{R}\left(\partial^{\lambda} \sigma \partial_{\lambda} \sigma\right)^{2}+\nabla^{\mu} \partial^{\nu} \sigma \nabla_{\mu} \partial_{\nu} \sigma \partial^{\lambda} \sigma \partial_{\lambda} \sigma-\left(\nabla^{2} \sigma\right)^{2} \partial^{\lambda} \sigma \partial_{\lambda} \sigma\right) \\
+ & 36 a \nabla^{2} \sigma\left(\partial^{\lambda} \sigma \partial_{\lambda} \sigma\right)^{2}+24 a\left(\partial^{\lambda} \sigma \partial_{\lambda} \sigma\right)^{3},
\end{aligned}
$$

which matches [12]. For the contributions arising from $L_{6}^{g}$ given by $(2.25)$ and $X_{6}^{g \mu \nu}$ given by $(2.23)$

$$
\begin{aligned}
\mathcal{L}_{6}^{g}(\sigma)= & \sigma L_{6}^{g}+\frac{1}{2} X_{6}^{g \mu \nu} \partial_{\mu} \sigma \partial_{\nu} \sigma \\
+ & 2 g_{I J} \partial^{\mu} g^{I} \partial^{\nu} g^{J}\left(6 \nabla^{\lambda} \partial_{\mu} \sigma \partial_{\nu} \sigma \partial_{\lambda} \sigma+\nabla_{\mu} \partial_{\nu} \sigma \partial^{\lambda} \sigma \partial_{\lambda} \sigma-2 \nabla^{2} \sigma \partial_{\mu} \sigma \partial_{\nu} \sigma\right. \\
& \left.\quad-4 \partial_{\mu} \sigma \partial_{\nu} \sigma \partial^{\lambda} \sigma \partial_{\lambda} \sigma\right) \\
& -g_{I J} \partial^{\lambda} g^{I} \partial_{\lambda} g^{J}\left(6 \nabla^{\mu} \partial^{\nu} \sigma \partial_{\mu} \sigma \partial_{\nu} \sigma+\nabla^{2} \sigma \partial^{\mu} \sigma \partial_{\mu} \sigma-\left(\partial^{\mu} \sigma \partial_{\mu} \sigma\right)^{2}\right) .
\end{aligned}
$$

The remaining contributions from (2.30) with (2.31) and (2.32) are then

$$
\mathcal{L}_{6}^{j}(\sigma)=\sigma L_{6}^{j}-\frac{1}{2} X_{6}^{j \mu \nu} \partial_{\mu} \sigma \partial_{\nu} \sigma, \quad \mathcal{L}_{6}^{k}(\sigma)=\sigma L_{6}^{k}
$$

\section{Fermions}

For completeness we extend the results in [20] to include background gauge fields coupled to fermion conserved currents $\bar{\psi} \gamma^{\mu} t_{a} \psi$. In this case the one loop action is determined by an operator $\Delta=-\not{D}^{2}$, with $\mathcal{D}_{\mu}$ including the spinor and gauge connections. This can be reduced to the form (3.9) where

$$
F_{\mu \nu} \rightarrow \frac{1}{4} R_{\mu \nu \lambda \rho} \gamma^{\lambda} \gamma^{\rho}+F_{\mu \nu} 1_{S}, \quad Y \rightarrow \frac{1}{2} \hat{R} 1_{S}-\frac{1}{2} F_{\mu \nu} \gamma^{\mu} \gamma^{\nu}
$$

with $1_{S}$ the spinor identity. For fermions then

$$
L_{6}^{R}+L_{6}^{F}=-\operatorname{tr}\left(a_{\Delta, 3} \mid\right)+\nabla_{\mu} \nabla_{\nu} Z^{\mu \nu},
$$


where the trace is over both spinorial and gauge indices. In the formula (3.11) for $\operatorname{tr}\left(a_{\Delta, 3} \mid\right)$ we may use (C.1) to obtain in six dimensions, using $\operatorname{tr}\left(1_{S}\right)=8$,

$$
\begin{aligned}
& \operatorname{tr}(\hat{I}) \rightarrow \frac{1}{3}\left(4 I_{1}-I_{2}-4 I_{3}\right)+20 P_{\mu \nu} W^{\mu \lambda \rho \omega} W_{\lambda \rho \omega}^{\nu}-6 \hat{R} W^{\mu \nu \lambda \rho} W_{\mu \nu \lambda \rho} \\
&-20 \hat{R} \nabla^{2} \hat{R}-112 P^{\mu \nu} P_{\nu \lambda} P_{\mu}^{\lambda}+56 \hat{R} P^{\mu \nu} P_{\mu \nu}+16 \hat{R}^{3} \\
&+8 \operatorname{tr}(\hat{I}), \\
& \operatorname{tr}\left(F^{\mu \nu} F_{\nu \lambda} F_{\mu}^{\lambda}\right) \rightarrow-I_{1}-3 P_{\mu \nu} W^{\mu \lambda \rho \omega} W_{\lambda \rho \omega}^{\nu}-6 P_{\mu \nu} P_{\lambda \rho} W^{\mu \lambda \rho \nu} \\
&-20 P^{\mu \nu} P_{\nu \lambda} P_{\mu}^{\lambda}+18 \hat{R} P^{\mu \nu} P_{\mu \nu}+2 \hat{R}^{3} \\
&+8 \operatorname{tr}\left(F^{\mu \nu} F_{\nu \lambda} F_{\mu}^{\lambda}\right), \\
& W_{\mu \nu \lambda \rho} \operatorname{tr}\left(F^{\mu \nu} F^{\lambda \rho}\right) \rightarrow-I_{2}-8 P_{\mu \nu} W^{\mu \lambda \rho \omega} W_{\lambda \rho \omega}^{\nu}+8 P_{\mu \nu} P_{\lambda \rho} W^{\mu \lambda \rho \nu} \\
&+8 W_{\mu \nu \lambda \rho} \operatorname{tr}\left(F^{\mu \nu} F^{\lambda \rho}\right) \\
& \operatorname{tr}\left(F^{\mu \nu} F_{\mu \nu} Y\right) \rightarrow-\frac{1}{2}\left(\hat{R} W^{\mu \nu \lambda \rho} W_{\mu \nu \lambda \rho}+16 \hat{R} P^{\mu \nu} P_{\mu \nu}+4 \hat{R}^{3}\right) \\
&+4 W_{\mu \nu \lambda \rho} \operatorname{tr}\left(F^{\mu \nu} F^{\lambda \rho}\right)+16 P_{\mu \nu} \operatorname{tr}\left(F^{\mu \lambda} F_{\lambda}^{\nu}\right)+4 \hat{R} \operatorname{tr}\left(F^{\mu \nu} F_{\mu \nu}\right), \\
& \operatorname{tr}\left(Y^{3}\right) \rightarrow \hat{R}^{3}-8 \operatorname{tr}\left(F^{\mu \nu} F_{\nu \lambda} F_{\mu}^{\lambda}\right)-6 \hat{R} \operatorname{tr}\left(F^{\mu \nu} F_{\mu \nu}\right), \\
& \operatorname{tr}\left(Y \nabla^{2} Y\right) \rightarrow 2 \hat{R} \nabla^{2} \hat{R}-4 \operatorname{tr}(\hat{I})+16 P_{\mu \nu} \operatorname{tr}\left(F^{\mu \lambda} F_{\lambda}^{\nu}\right)-16 \hat{R} \operatorname{tr}\left(F^{\mu \nu} F_{\mu \nu}\right),
\end{aligned}
$$

where on the right hand side the trace is only over gauge indices. To calculate the result for $\operatorname{tr}(\hat{I})$ it is necessary to use (A.20) and (A.24) to eliminate $P^{\mu \nu} \nabla^{2} P_{\mu \nu}$ with

$$
\begin{aligned}
16\left(P^{\mu \nu} \nabla^{2} P_{\mu \nu}-\hat{R} \nabla^{2} \hat{R}\right) \rightarrow & -\frac{1}{3}\left(4 I_{1}-I_{2}-I_{3}\right)-8 P_{\mu \nu} W^{\mu \lambda \rho \omega} W_{\lambda \rho \omega}^{\nu}+2 \hat{R} W^{\mu \nu \lambda \rho} W_{\mu \nu \lambda \rho} \\
& +16 P_{\mu \nu} P_{\lambda \rho} W^{\mu \lambda \rho \nu}+96 P^{\mu \nu} P_{\nu \lambda} P_{\mu}^{\lambda}-16 \hat{R} P^{\mu \nu} P_{\mu \nu},
\end{aligned}
$$

discarding two derivative terms. The traces in (C.3) give, for $n_{\psi}$ fermions, using from (A.10)

$$
\begin{aligned}
6 P_{\mu \nu} W^{\mu \lambda \rho \omega} W_{\lambda \rho \omega}^{\nu}= & 2 I_{1}+I_{2}-\frac{1}{4} E_{6}+\frac{3}{2} \hat{R} W_{\lambda \rho}^{\mu \nu} W_{\mu \nu}{ }^{\lambda \rho}-12 P_{\mu \nu} P_{\lambda \rho} W^{\mu \lambda \rho \nu} \\
& +24 P^{\mu \nu} P_{\nu \lambda} P_{\mu}^{\lambda}-36 \hat{R} P^{\mu \nu} P_{\mu \nu}+12 \hat{R}^{3}
\end{aligned}
$$

the result from (C.2)

$$
\begin{aligned}
L_{6}^{R} & =n_{\psi} \frac{1}{7 !}\left(-\frac{1}{3} 14 \times 64 I_{1}-32 I_{2}+40 I_{3}+\frac{191}{9} E_{6}\right), \\
L_{6}^{G} & =\operatorname{tr}\left(\frac{4}{15} \hat{I}+\frac{2}{9} W_{\mu \nu \lambda \rho} \operatorname{tr}\left(F^{\mu \nu} F^{\lambda \rho}\right)-\frac{52}{45} F^{\mu \nu} F_{\nu \lambda} F^{\lambda}{ }_{\mu}\right) .
\end{aligned}
$$

\section{Two-forms}

We here summarise some of the results necessary in the calculation of $a_{\Delta, 3} \mid$ for two-forms in (3.14).

$$
\operatorname{tr}_{\Omega^{(1)}}(\hat{I})=\frac{1}{4} \operatorname{tr}_{\Omega^{(2)}}(\hat{I})
$$




$$
\begin{aligned}
& \rightarrow-I_{3}-16 P^{\mu \nu} \nabla^{2} P_{\mu \nu}-4 \hat{R} \nabla^{2} \hat{R}+12 P_{\mu \nu} W^{\mu \lambda \rho \omega} W_{\lambda \rho \omega}^{\nu}-4 \hat{R} W^{\mu \nu \lambda \rho} W_{\mu \nu \lambda \rho} \\
&+16 P_{\mu \nu} P_{\lambda \rho} W^{\mu \lambda \rho \nu}-16 P^{\mu \nu} P_{\nu \lambda} P_{\mu}^{\lambda}+40 \hat{R} P^{\mu \nu} P_{\mu \nu}+16 \hat{R}^{3} \\
& \operatorname{tr}_{\Omega^{(1)}}\left(F^{\mu \nu} F_{\nu \lambda} F^{\lambda}\right)= \frac{1}{4} \operatorname{tr}_{\Omega^{(2)}}\left(F^{\mu \nu} F_{\nu \lambda} F_{\mu}^{\lambda}\right) \\
&=-I_{1}-3 P_{\mu \nu} W^{\mu \lambda \rho \omega} W_{\lambda \rho \omega}^{\nu}-6 P_{\mu \nu} P_{\lambda \rho} W^{\mu \lambda \rho \nu}-20 P^{\mu \nu} P_{\nu \lambda} P_{\mu}^{\lambda}+18 \hat{R} P^{\mu \nu} P_{\mu \nu}+2 \hat{R}^{3}, \\
& W_{\mu \nu \lambda \rho} \operatorname{tr}_{\Omega^{(1)}}\left(F^{\mu \nu} F^{\lambda \rho}\right)= \frac{1}{4} W_{\mu \nu \lambda \rho} \operatorname{tr}_{\Omega^{(2)}}\left(F^{\mu \nu} F^{\lambda \rho}\right) \\
&=-I_{2}-8 P_{\mu \nu} W^{\mu \lambda \rho \omega} W_{\lambda \rho \omega}^{\nu}+8 P_{\mu \nu} P_{\lambda \rho} W^{\mu \lambda \rho \nu}, \\
& \operatorname{tr}_{\Omega^{(1)}}\left(F^{\mu \nu} F_{\mu \nu} Y_{1}\right)=- 4 P_{\mu \nu} W^{\mu \lambda \rho \omega} W_{\lambda \rho \omega}^{\nu}+\hat{R} W^{\mu \nu \lambda \rho} W_{\mu \nu \lambda \rho}+16 P_{\mu \nu} P_{\lambda \rho} W^{\mu \lambda \rho \nu} \\
&-16 P^{\mu \nu} P_{\nu \lambda} P_{\mu}^{\lambda}-8 \hat{R} P^{\mu \nu} P_{\mu \nu}+4 \hat{R}^{3} \\
&-U_{\mu \nu} W^{\mu \lambda \rho \omega} W_{\lambda \rho \omega}^{\nu}-U W^{\mu \nu \lambda \rho} W_{\mu \nu \lambda \rho}+4 U_{\mu \nu} P_{\lambda \rho} W^{\mu \lambda \rho \nu} \\
&-4 P_{\mu}^{\nu} P_{\nu}^{\rho} U_{\rho}^{\mu}-2 P_{\mu}^{\nu} P_{\nu}^{\mu}\left(U_{\rho}^{\rho}+8 U\right)-4 \hat{R} P_{\mu}^{\nu} U_{\nu}^{\mu}-4 \hat{R}^{2} U, \\
& \operatorname{tr}_{\Omega^{(2)}}\left(F^{\mu \nu} F_{\mu \nu} Y_{2}\right)=-I_{2}-12 P_{\mu \nu} W^{\mu \lambda \rho \omega} W^{\nu}{ }_{\lambda \rho \omega}-2 \hat{R} W^{\mu \nu \lambda \rho} W_{\mu \nu \lambda \rho}+24 P_{\mu \nu} P_{\lambda \rho} W^{\mu \lambda \rho \nu} \\
&-16 P^{\mu \nu} P_{\nu \lambda} P_{\mu}^{\lambda}-56 \hat{R} P^{\mu \nu} P_{\mu \nu}-8 \hat{R}^{3} \\
&-2 U_{\mu \nu} W^{\mu \lambda \rho \omega} W_{\lambda \rho \omega}^{\nu}-\left(U_{\omega}^{\omega}+4 U\right) W^{\mu \nu \lambda \rho} W_{\mu \nu \lambda \rho}+8 U_{\mu \nu} P_{\lambda \rho} W^{\mu \lambda \rho \nu} \\
&-8 P_{\mu}^{\nu} P_{\nu}^{\rho} U_{\rho}^{\mu}-P_{\mu}^{\nu} P_{\nu}^{\mu}\left(20 U_{\rho}^{\rho}+64 U\right)-8 \hat{R} P_{\mu}^{\nu} U_{\nu}^{\mu}-4 \hat{R}^{2}\left(U_{\mu}^{\mu}+4 U\right),
\end{aligned}
$$

$\operatorname{tr}_{\Omega^{(0)}}\left(Y_{0}\right)=-2 \hat{R}+U, \quad \operatorname{tr}_{\Omega^{(1)}}\left(Y_{1}\right)=-2 \hat{R}+6 U+U_{\mu}^{\mu}, \quad \operatorname{tr}_{\Omega^{(2)}}\left(Y_{2}\right)=10 \hat{R}+15 U+5 U_{\mu}^{\mu}$,

$\operatorname{tr}_{\Omega^{(0)}}\left(Y_{0}^{2}\right)=(2 \hat{R}-U)^{2}$,

$\operatorname{tr}_{\Omega^{(1)}}\left(Y_{1}^{2}\right)=16 P^{\mu \nu} P_{\mu \nu}-2 \hat{R}^{2}+8 P_{\mu}^{\nu} U_{\nu}^{\mu}-2 \hat{R} U_{\mu}^{\mu}-4 \hat{R} U+U_{\mu}^{\nu} U_{\nu}^{\mu}+2 U U_{\mu}^{\mu}+6 U^{2}$,

$\operatorname{tr}_{\Omega^{(2)}}\left(Y_{2}^{2}\right)=W^{\mu \nu \lambda \rho} W_{\mu \nu \lambda \rho}+16 P^{\mu \nu} P_{\mu \nu}+4 \hat{R}^{2}+16 P_{\mu}^{\nu} U_{\nu}^{\mu}+4 \hat{R} U_{\mu}^{\mu}+20 \hat{R} U$

$$
+4 U_{\mu}^{\nu} U_{\nu}^{\mu}+U_{\mu}^{\mu} U_{\nu}^{\nu}+10 U U_{\mu}^{\mu}+15 U^{2}
$$

$\operatorname{tr}_{\Omega^{(0)}}\left(Y_{0}^{3}\right)=-(2 \hat{R}-U)^{3}$,

$\operatorname{tr}_{\Omega^{(1)}}\left(Y_{1}^{3}\right)=64 P^{\mu \nu} P_{\nu \lambda} P_{\mu}^{\lambda}-48 \hat{R} P^{\mu \nu} P_{\mu \nu}+6 \hat{R}^{3}$

$$
\begin{aligned}
& +48\left(P_{\mu}^{\nu} P_{\nu}^{\rho} U_{\rho}^{\mu}+P_{\mu}^{\nu} P_{\nu}^{\mu} U\right)-24 \hat{R} P_{\mu}^{\nu} U_{\nu}^{\mu}+3 \hat{R}^{2} U_{\mu}^{\mu}-6 \hat{R}^{2} U \\
& +12 P_{\mu}^{\nu} U_{\nu}^{\rho} U_{\rho}^{\mu}+24 P_{\mu}^{\nu} U_{\nu}^{\mu} U-3 \hat{R} U_{\mu}^{\nu} U_{\nu}^{\mu}-6 \hat{R}\left(U_{\mu}^{\mu} U+U^{2}\right) \\
& +U_{\mu}^{\nu} U_{\nu}^{\rho} U_{\rho}^{\mu}+3 U_{\mu}^{\nu} U_{\nu}^{\mu} U+3 U_{\mu}^{\mu} U^{2}+6 U^{3},
\end{aligned}
$$

$\operatorname{tr}_{\Omega^{(2)}}\left(Y_{2}^{3}\right)=-I_{2}+12 P_{\mu \nu} W^{\mu \lambda \rho \omega} W_{\lambda \rho \omega}^{\nu}+24 P_{\mu \nu} P_{\lambda \rho} W^{\mu \lambda \rho \nu}+16 P^{\mu \nu} P_{\nu \lambda} P_{\mu}^{\lambda}+24 \hat{R} P^{\mu \nu} P_{\mu \nu}$ $+6 U_{\mu \nu} W^{\mu \lambda \rho \omega} W_{\lambda \rho \omega}^{\nu}+3 U W^{\mu \nu \lambda \rho} W_{\mu \nu \lambda \rho}+24 U_{\mu \nu} P_{\lambda \rho} W^{\mu \lambda \rho \nu}+6 U_{\mu \nu} U_{\lambda \rho} W^{\mu \lambda \rho \nu}$

$+24 P_{\mu}^{\nu} P_{\nu}^{\rho} U_{\rho}^{\mu}+12 P_{\mu}^{\nu} P_{\nu}^{\mu}\left(U_{\rho}^{\rho}+4 U\right)+24 \hat{R} P_{\mu}^{\nu} U_{\nu}^{\mu}+12 \hat{R}^{2} U$

$+12 P_{\mu}^{\nu} U_{\nu}^{\rho} U_{\rho}^{\mu}+12 P_{\mu}^{\nu} U_{\nu}^{\mu}\left(U_{\rho}^{\rho}+4 U\right)+6 \hat{R} U_{\mu}^{\nu} U_{\nu}^{\mu}+12 \hat{R} U_{\mu}^{\mu} U+30 \hat{R} U^{2}$

$+2 U_{\mu}^{\nu} U_{\nu}^{\rho} U_{\rho}^{\mu}+3 U_{\mu}^{\nu} U_{\nu}^{\mu}\left(U_{\rho}^{\rho}+4 U\right)+3 U_{\mu}^{\mu} U_{\nu}^{\nu} U+15 U_{\mu}^{\mu} U^{2}+15 U^{3}$,

$\operatorname{tr}_{\Omega^{(0)}}\left(Y_{0} \nabla^{2} Y_{0}\right)=(2 \hat{R}-U) \nabla^{2}(2 \hat{R}-U)$,

$\operatorname{tr}_{\Omega^{(1)}}\left(Y_{1} \nabla^{2} Y_{1}\right)=16 P^{\mu \nu} \nabla^{2} P_{\mu \nu}-2 \hat{R} \nabla^{2} \hat{R}$

$$
\begin{aligned}
& +4\left(P^{\mu \nu} \nabla^{2} U_{\mu \nu}+U^{\mu \nu} \nabla^{2} P_{\mu \nu}\right)-U_{\mu}^{\mu} \nabla^{2} \hat{R}-\hat{R} \nabla^{2} U_{\mu}^{\mu}-2\left(U \nabla^{2} \hat{R}+\hat{R} \nabla^{2} U\right) \\
& +U^{\mu \nu} \nabla^{2} U_{\mu \nu}+U_{\mu}^{\mu} \nabla^{2} U+U \nabla^{2} U_{\mu}^{\mu}+6 U \nabla^{2} U
\end{aligned}
$$




$$
\begin{aligned}
\operatorname{tr}_{\Omega^{(2)}}\left(Y_{2} \nabla^{2} Y_{2}\right) & \rightarrow I_{3}+16 P^{\mu \nu} \nabla^{2} P_{\mu \nu}+4 \hat{R} \nabla^{2} \hat{R}-16 P_{\mu \nu} W^{\mu \lambda \rho \omega} W_{\lambda \rho \omega}^{\nu}+8 \hat{R} W^{\mu \nu \lambda \rho} W_{\mu \nu \lambda \rho} \\
& +8\left(P^{\mu \nu} \nabla^{2} U_{\mu \nu}+U^{\mu \nu} \nabla^{2} P_{\mu \nu}\right) \\
& +2\left(U_{\mu}^{\mu} \nabla^{2} \hat{R}+\hat{R} \nabla^{2} U_{\mu}^{\mu}\right)+10\left(U \nabla^{2} \hat{R}+\hat{R} \nabla^{2} U\right) \\
& +4 U^{\mu \nu} \nabla^{2} U_{\mu \nu}+U_{\mu}^{\mu} \nabla^{2} U_{\nu}^{\nu}+5\left(U_{\mu}^{\mu} \nabla^{2} U+U \nabla^{2} U_{\mu}^{\mu}\right)+15 U \nabla^{2} U .
\end{aligned}
$$

Combining terms as in (3.11) and using (C.4), (C.5) we find

$$
\begin{aligned}
& \operatorname{tr}_{\Omega^{(2)}}\left(a_{\Delta^{(2)}, 3} \mid\right)-2 \operatorname{tr}_{\Omega^{(1)}}\left(a_{\Delta^{(1)}, 3} \mid\right)+3 \operatorname{tr}_{\Omega^{(0)}}\left(a_{\Delta^{(0)}, 3} \mid\right) \\
& \rightarrow L_{6}^{R} \\
& \quad-G_{6}{ }^{\mu \nu} U_{\mu \nu}-\frac{11}{30} W^{\mu \nu \lambda \rho} W_{\mu \nu \lambda \rho} U^{\prime} \\
& \quad+\left(\frac{32}{3} P^{\mu \nu} P_{\mu \nu}-12 \hat{R}^{2}\right) U^{\prime}+\frac{2}{3}\left(\nabla^{2} \hat{R} U^{\prime}+\hat{R} \nabla^{2} U^{\prime}\right) \\
& \quad-U_{\mu \nu} U_{\lambda \rho} W^{\mu \lambda \rho \nu}+2 P^{\mu \nu} U_{\mu \rho} U_{\nu}^{\rho}-2 P^{\mu \nu} U_{\mu \nu} U_{\rho}^{\rho}-\frac{7}{3} \hat{R} U^{\mu \nu} U_{\mu \nu}+\frac{13}{12} \hat{R} U_{\mu}^{\mu} U_{\nu}^{\nu} \\
& \quad+\frac{1}{6}\left(U^{\mu \nu} \nabla^{2} U_{\mu \nu}-\frac{1}{4} U_{\mu}^{\mu} \nabla^{2} U_{\nu}^{\nu}\right) \\
& \quad-\left(U^{\mu \nu} U_{\mu \nu}-\frac{1}{4} U_{\mu}^{\mu} U_{\nu}^{\nu}\right) U^{\prime}-4 \hat{R} U^{\prime 2}-\frac{1}{2} U^{\prime} \Delta_{2} U^{\prime}-U^{\prime 3},
\end{aligned}
$$

where $L_{6}^{R}$ is given in (3.15) and

$$
U^{\prime}=U+\frac{1}{2} U_{\mu}^{\mu}
$$

From (3.13) $U^{\prime}=\frac{1}{4} v^{\mu} v_{\mu}, U_{\mu \nu}=-\nabla_{\mu} v_{\nu}=\nabla_{\mu} \partial_{\nu} \ln g^{2}$. Since $G_{6}{ }^{\mu \nu} U_{\mu \nu}=\nabla_{\mu} \nabla_{\nu}\left(G_{6}{ }^{\mu \nu} \ln g^{2}\right)$ this term may be neglected and (D.1) leads to (3.16) using

$$
\begin{aligned}
\nabla_{\mu} v_{\nu} \nabla_{\lambda} v_{\rho} W^{\mu \lambda \rho \nu} \rightarrow & -\left(\frac{1}{2} W^{\mu \lambda \rho \omega} W_{\lambda \rho \omega}^{\nu}-4 P_{\lambda \rho} W^{\mu \lambda \rho \nu}-3 B^{\mu \nu}\right) v_{\mu} v_{\nu} \\
= & -\left(\frac{1}{2} W^{\mu \lambda \rho \omega} W_{\lambda \rho \omega}^{\nu}-B^{\mu \nu}+12 P^{\mu \lambda} P_{\lambda}^{\nu}-2 \nabla^{2} P^{\mu \nu}+2 \nabla^{\mu} \partial^{\nu} \hat{R}\right) v_{\mu} v_{\nu} \\
& +2 P^{\lambda \rho} P_{\lambda \rho} v^{\mu} v_{\mu}, \\
\hat{R} \nabla^{\mu} v^{\nu} \nabla_{\mu} v_{\nu} \rightarrow & \hat{R} \nabla_{\mu} v^{\mu} \nabla_{\nu} v^{\nu}-\left(4 \hat{R} P^{\mu \nu}+\nabla^{\mu} \partial^{\nu} \hat{R}\right) v_{\mu} v_{\nu}-\left(\hat{R}^{2}-\nabla^{2} \hat{R}\right) v^{\mu} v_{\mu}, \\
P^{\mu \nu} \nabla_{\mu} v^{\lambda} \nabla_{\nu} v_{\lambda} \rightarrow & P^{\mu \nu} \nabla_{\mu} v_{\nu} \nabla_{\lambda} v^{\lambda}-\left(4 P^{\mu \lambda} P_{\lambda}^{\nu}+\hat{R} P^{\mu \nu}-\frac{1}{2} \nabla^{2} P^{\mu \nu}+\nabla^{\mu} \partial^{\nu} \hat{R}\right) v_{\mu} v_{\nu} \\
& +\frac{1}{2} \nabla^{2} \hat{R} v^{\mu} v_{\mu}, \quad \\
\nabla^{\mu} v^{\nu} \nabla^{2} \nabla_{\mu} v_{\nu} \rightarrow & \nabla_{\mu} v^{\mu} \nabla^{2} \nabla_{\nu} v^{\nu}+12 P^{\mu \nu} \nabla_{\mu} v_{\nu} \nabla_{\lambda} v^{\lambda}+3 \hat{R} \nabla_{\mu} v^{\mu} \nabla_{\nu} v^{\nu} \\
& +\left(-W^{\mu \lambda \rho \omega} W_{\lambda \rho \omega}^{\nu}-60 P^{\mu \lambda} P_{\lambda}^{\nu}-20 \hat{R} P^{\mu \nu}\right. \\
& +\left(2 P^{\lambda \rho} P_{\lambda \rho}-2 \hat{R}^{2}+9 \nabla^{2} \hat{R}\right) v^{\mu} v_{\mu},
\end{aligned}
$$

discarding total derivatives. 
Open Access. This article is distributed under the terms of the Creative Commons Attribution License (CC-BY 4.0), which permits any use, distribution and reproduction in any medium, provided the original author(s) and source are credited.

\section{References}

[1] D. Green, Z. Komargodski, N. Seiberg, Y. Tachikawa and B. Wecht, Exactly marginal deformations and global symmetries, JHEP 06 (2010) 106 [arXiv:1005.3546] [INSPIRE].

[2] M. Henningson, A class of six-dimensional conformal field theories, Phys. Rev. Lett. 85 (2000) 5280 [hep-th/0006231] [INSPIRE].

[3] Z. Komargodski and A. Schwimmer, On renormalization group flows in four dimensions, JHEP 12 (2011) 099 [arXiv:1107.3987] [INSPIRE].

[4] M.A. Luty, J. Polchinski and R. Rattazzi, The a-theorem and the asymptotics of $4 D$ quantum field theory, JHEP 01 (2013) 152 [arXiv: 1204.5221] [INSPIRE].

[5] A.B. Zamolodchikov, Irreversibility of the flux of the renormalization group in a $2 D$ field theory, JETP Lett. 43 (1986) 730 [Pisma Zh. Eksp. Teor. Fiz. 43 (1986) 565] [InSPIRE].

[6] H. Osborn, Weyl consistency conditions and a local renormalization group equation for general renormalizable field theories, Nucl. Phys. B 363 (1991) 486 [INSPIRE].

[7] I. Jack and H. Osborn, Constraints on RG flow for four dimensional quantum field theories, Nucl. Phys. B 883 (2014) 425 [arXiv:1312.0428] [inSPIRE].

[8] F. Baume, B. Keren-Zur, R. Rattazzi and L. Vitale, The local Callan-Symanzik equation: structure and applications, JHEP 08 (2014) 152 [arXiv:1401.5983] [INSPIRE].

[9] I. Jack and H. Osborn, Analogs for the $c$ theorem for four-dimensional renormalizable field theories, Nucl. Phys. B 343 (1990) 647 [InSPIRE].

[10] J.-F. Fortin, B. Grinstein and A. Stergiou, Scale without conformal invariance at three loops, JHEP 08 (2012) 085 [arXiv: 1202.4757] [inSPIRE].

[11] J.-F. Fortin, B. Grinstein and A. Stergiou, Limit cycles and conformal invariance, JHEP 01 (2013) 184 [arXiv: 1208.3674] [INSPIRE].

[12] H. Elvang et al., On renormalization group flows and the a-theorem in $6 D$, JHEP 10 (2012) 011 [arXiv: 1205. 3994] [INSPIRE].

[13] H. Elvang and T.M. Olson, $R G$ flows in d dimensions, the dilaton effective action and the a-theorem, JHEP 03 (2013) 034 [arXiv:1209.3424] [INSPIRE].

[14] F. Baume and B. Keren-Zur, The dilaton Wess-Zumino action in higher dimensions, JHEP 11 (2013) 102 [arXiv:1307.0484] [INSPIRE].

[15] C. Corianò, L. Delle Rose, C. Marzo and M. Serino, The dilaton Wess-Zumino action in six dimensions from Weyl gauging: local anomalies and trace relations, Class. Quant. Grav. 31 (2014) 105009 [arXiv:1311.1804] [InSPIRE].

[16] B. Grinstein, A. Stergiou and D. Stone, Consequences of Weyl consistency conditions, JHEP 11 (2013) 195 [arXiv:1308.1096] [inSPIRE].

[17] B. Grinstein, D. Stone, A. Stergiou and M. Zhong, Challenge to the a-theorem in six dimensions, Phys. Rev. Lett. 113 (2014) 231602 [arXiv:1406.3626] [INSPIRE]. 
[18] L. Bonora, P. Pasti and M. Bregola, Weyl cocycles, Class. Quant. Grav. 3 (1986) 635 [INSPIRE].

[19] S. Deser and A. Schwimmer, Geometric classification of conformal anomalies in arbitrary dimensions, Phys. Lett. B 309 (1993) 279 [hep-th/9302047] [INSPIRE].

[20] F. Bastianelli, S. Frolov and A.A. Tseytlin, Conformal anomaly of $(2,0)$ tensor multiplet in six-dimensions and AdS/CFT correspondence, JHEP 02 (2000) 013 [hep-th/0001041] [INSPIRE].

[21] D.M. Hofman and J. Maldacena, Conformal collider physics: energy and charge correlations, JHEP 05 (2008) 012 [arXiv:0803.1467] [INSPIRE].

[22] C. Fefferman and C.R. Graham, Conformal invariants, Astérique, Hors Série 95, France (1985).

[23] C. Fefferman and C.R. Graham, The ambient metric, arXiv:0710.0919 [INSPIRE].

[24] H. Osborn, Local couplings and $\mathrm{SL}(2, R)$ invariance for gauge theories at one loop, Phys. Lett. B 561 (2003) 174 [hep-th/0302119] [INSPIRE].

[25] M.E. Fisher, Yang-Lee edge singularity and $\phi^{3}$ field theory, Phys. Rev. Lett. 40 (1978) 1610 [INSPIRE].

[26] I. Jack, Renormalizability of $\phi^{3}$ theory in six-dimensional curved space-time, Nucl. Phys. B 274 (1986) 139 [InSPIRE].

[27] J. Kodaira, Interacting scalar field theory in general curved space-time, Phys. Rev. D 33 (1986) 2882 [inSPIRE].

[28] A.J. McKane, D.J. Wallace and R.K.P. Zia, Models for strong interactions in six epsilon dimensions, Phys. Lett. B 65 (1976) 171 [InSPIRE].

[29] A.J. Mckane, An $\mathrm{SU}(3) \times \mathrm{SU}(3)$ field theory of strong interactions in six epsilon dimensions, J. Phys. G 3 (1977) 1165 [inSPIRE].

[30] O.F. de Alcantara Bonfim, J.E. Kirkham and A.J. McKane, Critical exponents for the percolation problem and the Yang-Lee edge singularity, J. Phys. A 14 (1981) 2391 [INSPIRE].

[31] A.J. Mckane, Vacuum instability in scalar field theories, Nucl. Phys. B 152 (1979) 166 [INSPIRE].

[32] L. Fei, S. Giombi, I.R. Klebanov and G. Tarnopolsky, Three loop analysis of the critical $O(N)$ models in six epsilon dimensions, Phys. Rev. D 91 (2015) 045011 [arXiv:1411.1099] [INSPIRE].

[33] L.S. Brown and J.C. Collins, Dimensional renormalization of scalar field theory in curved space-time, Annals Phys. 130 (1980) 215 [INSPIRE].

[34] D.Z. Freedman, K. Johnson and J.I. Latorre, Differential regularization and renormalization: a new method of calculation in quantum field theory, Nucl. Phys. B 371 (1992) 353 [INSPIRE].

[35] H. Osborn and A.C. Petkou, Implications of conformal invariance in field theories for general dimensions, Annals Phys. 231 (1994) 311 [hep-th/9307010] [INSPIRE].

[36] A. Buchel et al., Holographic GB gravity in arbitrary dimensions, JHEP 03 (2010) 111 [arXiv: 0911.4257] [INSPIRE]. 
[37] J. de Boer, M. Kulaxizi and A. Parnachev, $A d S_{7} / C F T_{6}$, Gauss-Bonnet gravity and viscosity bound, JHEP 03 (2010) 087 [arXiv: 0910.5347] [INSPIRE].

[38] D.Z. Freedman, S.S. Gubser, K. Pilch and N.P. Warner, Renormalization group flows from holography supersymmetry and a c-theorem, Adv. Theor. Math. Phys. 3 (1999) 363 [hep-th/9904017] [INSPIRE].

[39] R.C. Myers and A. Sinha, Seeing a c-theorem with holography, Phys. Rev. D 82 (2010) 046006 [arXiv: 1006.1263] [InSPIRE].

[40] R.C. Myers and A. Sinha, Holographic c-theorems in arbitrary dimensions, JHEP 01 (2011) 125 [arXiv: 1011.5819] [InSPIRE].

[41] K. Yonekura, Perturbative c-theorem in d-dimensions, JHEP 04 (2013) 011 [arXiv: 1212.3028] [INSPIRE].

[42] S. Forte and J.I. Latorre, A proof of the irreversibility of renormalization group flows in four-dimensions, Nucl. Phys. B 535 (1998) 709 [hep-th/9805015] [INSPIRE].

[43] T. Parker and S. Rosenberg, Invariants of conformal laplacians,

J. Diff. Geom. 25 (1987) 199.

[44] J. Erdmenger, Conformally covariant differential operators: properties and applications, Class. Quant. Grav. 14 (1997) 2061 [hep-th/9704108] [INSPIRE].

[45] S.M. Paneitz, A quartic conformally covariant differential operator for arbitrary pseudo-Riemannian manifolds (summary), SIGMA 4 (2008) 36 [arXiv:0803.4331].

[46] E.S. Fradkin and A.A. Tseytlin, Asymptotic freedom in extended conformal supergravities, Phys. Lett. B 110 (1982) 117 [INSPIRE].

[47] E.S. Fradkin and A.A. Tseytlin, One loop $\beta$-function in conformal supergravities, Nucl. Phys. B 203 (1982) 157 [InSPIRE].

[48] E.S. Fradkin and A.A. Tseytlin, Conformal anomaly in Weyl theory and anomaly free superconformal theories, Phys. Lett. B 134 (1984) 187 [INSPIRE].

[49] R.J. Riegert, A nonlocal action for the trace anomaly, Phys. Lett. B 134 (1984) 56 [INSPIRE].

[50] T.P. Branson, Differential operators canonically associated to a conformal structure, Math. Scand. 57 (1985) 293.

[51] A.R. Gover and L.J. Peterson, Conformally invariant powers of the laplacian, Q-curvature and tractor calculus, Commun. Math. Phys. 235 (2003) 339 [math-ph/0201030] [INSPIRE].

[52] V. Wünsch, On conformally invariant differential operators, Math. Nach. 129 (1986) 269.

[53] J. Erdmenger and H. Osborn, Conformally covariant differential operators: symmetric tensor fields, Class. Quant. Grav. 15 (1998) 273 [gr-qc/9708040] [INSPIRE].

[54] P.B. Gilkey, The spectral geometry of a Riemannian manifold, J. Diff. Geom. 10 (1975) 601 [INSPIRE]. 